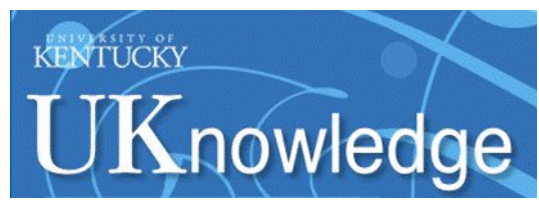

University of Kentucky

UKnowledge

\title{
THE EFFECTS OF CURCUMIN AND FENUGREEK SOLUBLE FIBER ON THE PHYSICAL WORKING CAPACITY AT THE FATIGUE THRESHOLD, PEAK OXYGEN CONSUMPTION, AND TIME TO EXHAUSTION
}

Lauren Herrick

University of Kentucky, lauren.herrick3@gmail.com

Author ORCID Identifier:

(iD) https://orcid.org/0000-0001-9116-5051

Digital Object Identifier: https://doi.org/10.13023/etd.2019.455

Right click to open a feedback form in a new tab to let us know how this document benefits you.

\section{Recommended Citation}

Herrick, Lauren, "THE EFFECTS OF CURCUMIN AND FENUGREEK SOLUBLE FIBER ON THE PHYSICAL WORKING CAPACITY AT THE FATIGUE THRESHOLD, PEAK OXYGEN CONSUMPTION, AND TIME TO EXHAUSTION" (2019). Theses and Dissertations--Kinesiology and Health Promotion. 68.

https://uknowledge.uky.edu/khp_etds/68

This Master's Thesis is brought to you for free and open access by the Kinesiology and Health Promotion at UKnowledge. It has been accepted for inclusion in Theses and Dissertations--Kinesiology and Health Promotion by an authorized administrator of UKnowledge. For more information, please contact UKnowledge@lsv.uky.edu. 


\section{STUDENT AGREEMENT:}

I represent that my thesis or dissertation and abstract are my original work. Proper attribution has been given to all outside sources. I understand that I am solely responsible for obtaining any needed copyright permissions. I have obtained needed written permission statement(s) from the owner(s) of each third-party copyrighted matter to be included in my work, allowing electronic distribution (if such use is not permitted by the fair use doctrine) which will be submitted to UKnowledge as Additional File.

I hereby grant to The University of Kentucky and its agents the irrevocable, non-exclusive, and royalty-free license to archive and make accessible my work in whole or in part in all forms of media, now or hereafter known. I agree that the document mentioned above may be made available immediately for worldwide access unless an embargo applies.

I retain all other ownership rights to the copyright of my work. I also retain the right to use in future works (such as articles or books) all or part of my work. I understand that I am free to register the copyright to my work.

\section{REVIEW, APPROVAL AND ACCEPTANCE}

The document mentioned above has been reviewed and accepted by the student's advisor, on behalf of the advisory committee, and by the Director of Graduate Studies (DGS), on behalf of the program; we verify that this is the final, approved version of the student's thesis including all changes required by the advisory committee. The undersigned agree to abide by the statements above.

Lauren Herrick, Student

Dr. Haley Bergstrom, Major Professor

Dr. Melinda Ickes, Director of Graduate Studies 
THE EFFECTS OF CURCUMIN AND FENUGREEK SOLUBLE FIBER ON THE PHYSICAL WORKING CAPACITY AT THE FATIGUE THRESHOLD, PEAK OXYGEN CONSUMPTION, AND TIME TO EXHAUSTION

\section{THESIS}

A thesis submitted in partial fulfillment of the

requirements for the degree of Master of Science in the

College of Education

at the University of Kentucky

By

Lauren Herrick

Lexington, Kentucky

Director: Dr. Haley Bergstrom, Assistant Professor of Kinesiology and Health Promotion

Lexington, Kentucky

2019

Copyright (C) Lauren Herrick 2019

https://orcid.org/0000-0001-9116-5051 


\section{ABSTRACT OF THESIS}

\section{THE EFFECTS OF CURCUMIN AND FENUGREEK SOLUBLE FIBER ON THE PHYSICAL WORKING CAPACITY AT THE FATIGUE THRESHOLD, PEAK OXYGEN CONSUMPTION, AND TIME TO EXHAUSTION}

Curcumin, a polyphenol, has been suggested to improve metabolic byproduct clearance and increase nitric oxide production in working muscle. These purported effects may delay neuromuscular fatigue. Therefore, the purpose of this study was to examine the effects of curcumin in combination with fenugreek (CUR) or fenugreek soluble fiber alone $(\mathrm{FEN})$ on the neuromuscular fatigue threshold $\left(\mathrm{PWC}_{\mathrm{FT}}\right)$, time to exhaustion ( $\left.\mathrm{T}_{\mathrm{lim}}\right)$ on a graded exercise test $(\mathrm{GXT})$, and $\dot{V} \mathrm{O}_{2}$ peak in untrained subjects. Forty-seven, college-aged, aerobically untrained individuals were randomly assigned to one of three supplementation groups; placebo (PLA, n=15), curcumin + fenugreek, CurQfen ${ }^{\circledR}(\mathrm{CUR}, \mathrm{n}=18)$, or fenugreek soluble fiber $(\mathrm{FEN}, \mathrm{n}=14)$. All subjects performed a maximal GXT on a cycle ergometer to determine the $\mathrm{PWC}_{\mathrm{FT}}, \mathrm{T}_{\text {lim }}$, and $\dot{\mathrm{VO}}_{2}$ peak before (PRE-test) and after (POST-test) 28 days of daily supplementation. Statistical analyses included 3 separate, one-way ANCOVAs to determine if there were any differences among the groups (PLA, CUR, FEN) for adjusted post-test scores for the $\mathrm{PWC}_{\mathrm{FT}}$, $\dot{\mathrm{V}} \mathrm{O}_{2}$ peak, and $\mathrm{T}_{\mathrm{lim}}$. The respective pre-test score was used as the covariate. In addition, reliability analyses (PRE- to POST-test) for the PLA group were used to calculate the minimal difference needed to be real (MD). The adjusted POST PWC $\mathrm{FT}_{\text {values showed }}$ no statistical differences between groups $(\mathrm{F}=3.141 \mathrm{p}=0.053)$; however pairwise LSD 
comparisons indicated a significant difference between the CUR and PLA groups ( $p=$ 0.016), but not between the CUR and FEN groups. Therefore, separate one-way ANCOVAs were used to examine the adjusted $\mathrm{PWC}_{\mathrm{FT}}$ means for the PLA vs. CUR ( $\mathrm{F}=$ 4.906, $\mathrm{p}=0.035)$ and the PLA vs. FEN $(\mathrm{F}=2.969, \mathrm{p}=0.097)$. The one- way ANCOVA for $\dot{V} \mathrm{O}_{2}$ peak $(\mathrm{F}=0.612 \mathrm{p}=0.547)$ and $\mathrm{T}_{\lim }(\mathrm{F}=0.688, \mathrm{p}=0.508)$ values showed no statistical difference among the groups. Individual responses in each group showed $20 \%$ of subjects in the CUR group, $\sim 7 \%$ in the FEN group, and $\sim 6 \%$ in the PLA group had values greater than the $\mathrm{MD}$ for the $\mathrm{PWC}_{\mathrm{FT}}$, but none of the subjects in the PLA, FEN, or CUR groups exceeded the MD for $\dot{V} \mathrm{O}_{2}$ peak or $\mathrm{T}_{\text {lim. }}$. These findings indicated CurQfen® supplementation increased the $\mathrm{PWC}_{\mathrm{FT}}$ compared to a placebo, but not compared to fenugreek soluble fiber alone. However, there were no effects of CurQfen $\AA$ on $\mathrm{V}_{2}$ peak or $\mathrm{T}_{\mathrm{lim}}$. The mechanisms responsible for delaying time to neuromuscular fatigue may include increased NO production and increased blood flow to remove metabolic byproducts; however, the cellular changes which could lead to increases in $\mathrm{T}_{\text {lim }}$ and $\dot{V} \mathrm{O}_{2}$ peak may not have been sensitive to the GXT protocol or the given dosage of curcumin supplementation. Considering individual responses, CurQfen ${ }^{\circledR}$ supplementation resulted in a real change in the $\mathrm{PWC}_{\mathrm{FT}}$ for a small portion of the subjects ( $\sim 20 \%)$. These findings suggested that CurQfen $®$ supplementation without exercise training may help to improve time to neuromuscular fatigue in certain individuals, but the group mean analyses were not necessarily reflective of the responses for a majority of the subjects. 


\begin{tabular}{c} 
Lauren Herrick \\
\hline (Name of Student) \\
$11 / 21 / 19$ \\
Date
\end{tabular}


THE EFFECTS OF CURCUMIN AND FENUGREEK SOLUBLE FIBER ON THE PHYSICAL WORKINGCAPACITY AT THE FATIGUE THRESHOLD, PEAK OXYGEN CONSUMPTION, AND TIME TO EXHAUSTION

By

Lauren Herrick

Haley Bergstrom

Director of Thesis

Melinda Ickes

Director of Graduate Studies

$11 / 21 / 19$

Date 


\section{DEDICATION}

To my parents Stephanie and David Herrick, and Grandpa Bob. 


\section{ACKNOWLEDGMENTS}

The following thesis, while an individual work, benefited greatly from the insights, direction and contributions of several people. First, my Thesis Chair, Haley Bergstrom, exemplifies the highest quality scholarship to which I aspire. Her unwavering support and encouragement as well as guidance during this project is unmatched, and I could not have done it without her. In addition, I want to thank my research partner Jensen Goh, who worked alongside me to conduct this study, for his time and positive attitude throughout, as well as Walter Menke for his help with subject testing. Next, I wish to thank my Thesis Committee, Mark Abel, Marilyn Campbell, and Bradley Fleenor for their time and commitment to my study. Each individual provided insights that guided and challenged my thinking, substantially improving the finished product. I would like to give additional thanks to Marilyn Campbell for her help in

obtaining the supplement for this investigation and Bradley Fleenor for his contribution to developing an applied study.

In addition to the technical and instrumental assistance above, I would like to thank my friends and family who provided on-going support throughout the thesis process. Finally, I wish to thank the respondents of my study (who remain anonymous for confidentiality purposes). 


\section{TABLE OFCONTENTS}

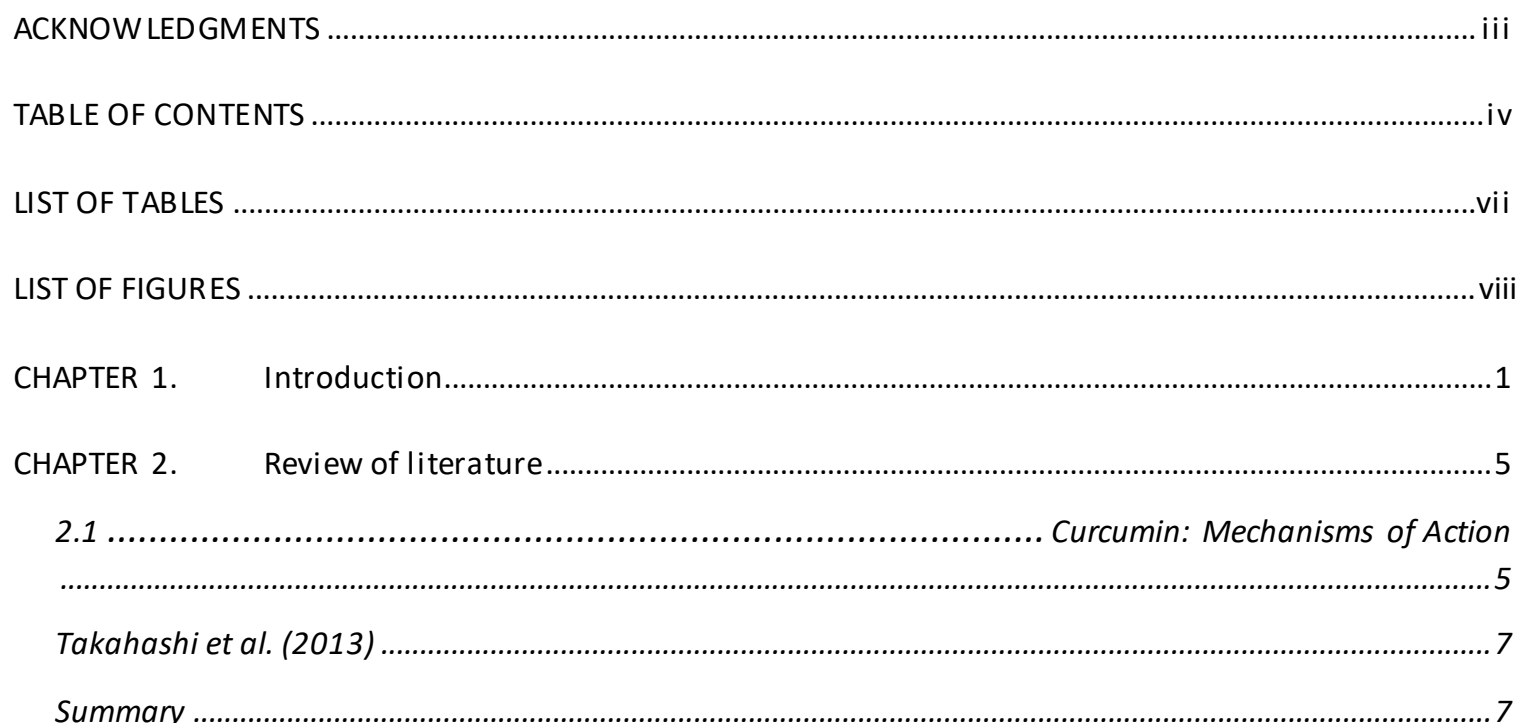

2.2 Physiological and Performance Effects of Curcumin supplementation

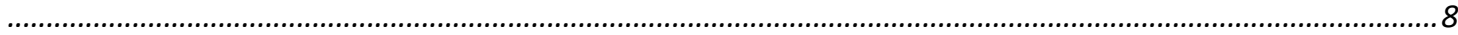

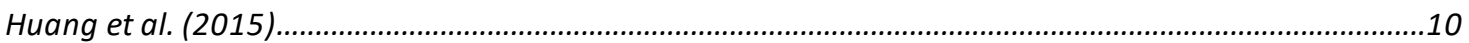

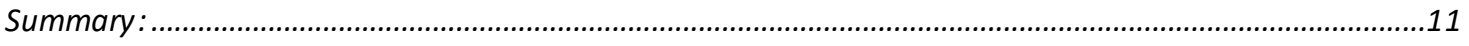

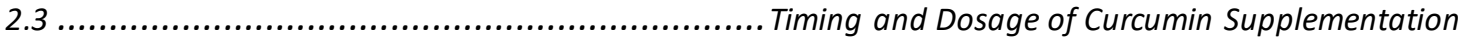

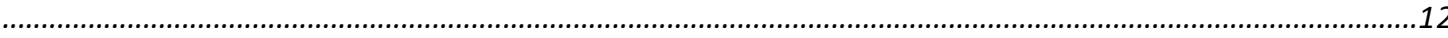

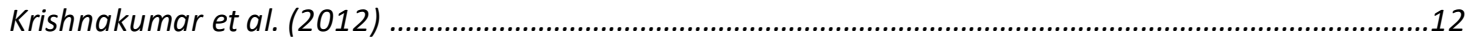

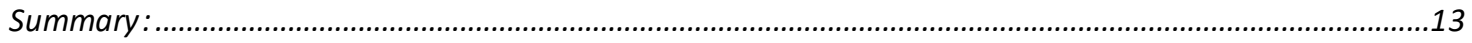

$2.4 \ldots . . . . . . . . . . . . . . . . .$. Development of the Physical Working Capacity at the Fatigue Threshold Model

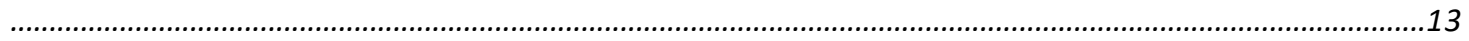

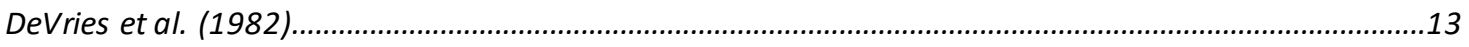

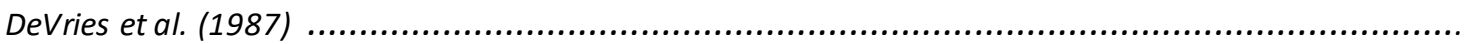

15

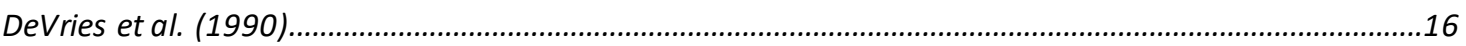

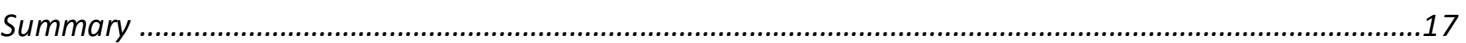

2.5 ...... Utilization of PWC FT to Examine the Efficacy of Nutritional Supplements to Improve Performance

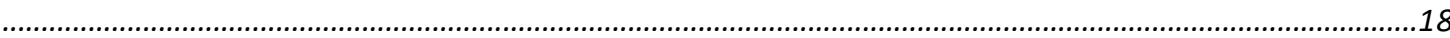

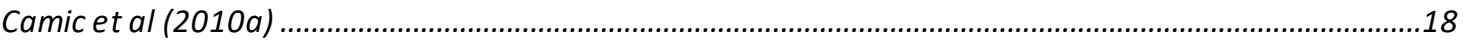

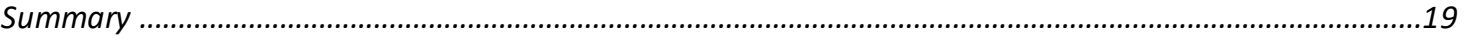

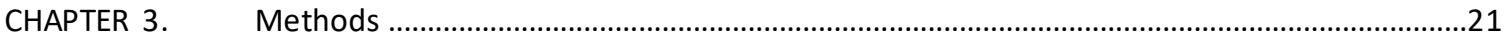


Table 1. Demographic information and PRE test values for time to exhaustion $\left(T_{\text {lim }}\right), V_{2} \mathrm{O}_{2}$ peak, and the physical working capacity at the fatigue threshold (PWC FT).

Table 2. Results of the reliability analyses for the placebo group using PRE test and POST test values for the physical working capacity at the fatigue threshold ( $\left.P W C_{F T}\right), V O_{2}$ peak, and time to exhaustion ( $\left.T_{\text {lim }}\right)$.

4.2 Mean Group Comparisons

Figure 1. Adjusted POST PWC FT (mean \pm SEM) values (covaried for PRE PWCFT SCores) for FEN, and PLA groups.

Figure 2. Adjusted POST PWC FT (mean \pm SEM) values (covaried for PRE PWC FT Scores) for CUR, and PLA groups *significantly $(p<0.05)$ greater than placebo.

Figure 3. Adjusted POST $V O_{2}$ peak (mean \pm SEM) values (covaried for PRE VO $O_{2}$ scores) for CUR, FEN, and PLA groups *significantly $(p<0.05)$ greater than placebo

Figure 4. Adjusted POST Tlim (mean \pm SEM) values (covaried for PRE Tlim scores) for CUR, FEN, and PLA groups *significantly $(p<0.05)$ greater than placebo

4.3 Individual Responses

Figure 5. Individual responses for the PWC from PRE- to POST-test for the CUR supplement group. Solid line indicates the subject had an increase greater than the minimal difference. Dashed line (---) = no change, dashed and dotted line (-. -) = decrease, and dotted line (...) = increase. Note there were 4 subjects who had increases grea ter than MD, two solid subject lines overlap at 155 - 245W..

Figure 6. Individual responses for the PWC from PRE- to POST-test for the FEN supplement group. Solid line indicates the subject had an increase greater than the minimal difference. Dashed line (---) = no change, dashed and dotted line (- . -) = decrease, and dotted line (...) = increase........ 
Figure 7. Individual responses for the PWC from PRE- to POST-test for the PLA supplement group. Solid line indicates the subject had an increase greater than the minimal difference, dashed, double dot, dashed (- . .) line indicates the subject had a decrease greater than the minimal difference. Dashed line $(---)=$ no change, dashed and dotted line (- . -) = decrease, and dotted line (...) = increase.

Figure 8. Individual responses for $\mathrm{VO}_{2}$ peak from PRE- to POST-test for the CUR supplement group. Solid line indicates the subject had an increase greater than the minimal difference, dashed, double dot, dashed (- . -) line indicates the subject had a decrease greater than the minimal difference. Dashed line $(--)$ = no change, dashed and dotted line (- . -) = decrease, and dotted line (...) = increase.

Figure 9. Individual responses for $\mathrm{VO}_{2}$ peak from PRE- to POST-test for the FEN supplement group. Solid line indicates the subject had an increase greater than the minimal difference, dashed, double dot, dashed (- . -) line indicates the subject had a decrease greater than the minimal difference. Dashed line $(---)=$ no change, dashed and dotted line (- . -) = decrease, and dotted line (...) = increase. 34

Figure 10. Individual responses for $\mathrm{VO}_{2}$ peakfrom PRE- to POST-test for the PLA supplement group. Solid line indicates the subject had an increase greater than the minimal difference, dashed, double dot, dashed (- . -) line indicates the subject had a decrease greater than the minimal difference. Dashed line $(---)=$ no change, dashed and dotted line (- . -) = decrease, and dotted line (...) = increase.

Figure 11. Individual responses for time to exhaustion from PRE-to POST-test for the CUR supplement group. Solid line indicates the subject had an increase greater than the minimal difference, dashed, double dot, dashed (- . -) line indicates the subject had a decrease greater than the minimal difference. Dashed line (---) = no change, dashed and dotted line (- . -) = decrease, and dotted line (...) = increase. 35

Figure 12. Individual responses for time to exhaustion from PRE- to POST-test for the FEN supplement group. Solid line indicates the subject had an increase greater than the minimal difference, dashed, double dot, dashed (- . -) line indicates the subject had a decrease greater than the minimal difference. Dashed line (---) = no change, dashed and dotted line (- . -) = decrease, and dotted line (...) = increase. 36

Figure 13. Individual responses for time to exhaustion from PRE- to POST-test for the PLA supplement group. Solid line indicates the subject had an increase greater than the minimal difference, dashed, double dot, dashed (- . .) line indicates the subject had a decrease greater than the minimal difference. Dashed line (---) = no change, dashed and dotted line (- . -) = decrease, and dotted line (...) = increase. 36

CHAPTER 5. Discussion

VITA 


\section{LIST OF TABLES}

Table 1. Demographic information and PRE test values for time to exhaustion $\left(T_{\text {lim }}\right), V_{2}$ peak, and the physical working capacity at the fatigue threshold (PWC FT) .....................................................................27

Table 2. Results of the reliability analyses for the placebo group using PRE test and POST test values for the physical working capacity at the fatigue threshold (PWC FT), VO $O_{2}$ peak, and time to exhaustion ( $\left.T_{\text {lim }}\right)$. 


\section{LIST OF FIGURES}

Figure 1. Adjusted POST PWC $F$ (mean \pm SEM) values (covaried for PRE PWC FT Scores) for FEN, and PLA

groups.

Figure 2. Adjusted POST PWC $F$ (mean \pm SEM) values (covaried for PRE PWC FT SCores) for CUR, and PLA groups *significantly $(p<0.05)$ greater than placebo.

Figure 3. Adjusted POST $\mathrm{VO}_{2}$ peak (mean \pm SEM) values (covaried for PRE $\mathrm{VO}_{2}$ scores) for CUR, FEN, and PLA groups *significantly $(p<0.05)$ greater than placebo

Figure 4. Adjusted POST Tlim (mean \pm SEM) values (covaried for PRE Tlim scores) for CUR, FEN, and PLA groups *significantly $(p<0.05)$ greater than placebo

Figure 5. Individual responses for the PWC from PRE- to POST-test for the CUR supplement group. Solid line indicates the subject had an increase greater than the minimal difference. Dashed line (---) = no change, dashed and dotted line (-. -) = decrease, and dotted line (...) = increase. Note there were 4 subjects who had increases greater than MD, two solid subject lines overlap at $155-245 W$..

Figure 6. Individual responses for the PWCFT from PRE- to POST-test for the FEN supplement group. Solid line indicates the subject had an increase greater than the minimal difference. Dashed line (---) =no change, dashed and dotted line (- . -) = decrease, and dotted line (...) = increase.....

Figure 7. Individual responses for the PWC from PRE- to POST-test for the PLA supplement group. Solid line indicates the subject had an increase greater than the minimal difference, dashed, double dot, dashed (- . -) line indicates the subject had a decrease greater than the minimal difference. Dashed line $(--)=$ no change, dashed and dotted line (- . -) = decrease, and dotted line (...) = increase.......

Figure 8. Individual responses for $\mathrm{VO}_{2}$ peak from PRE- to POST-test for the CUR supplement group. Solid line indicates the subject had an increase greater than the minimal difference, dashed, double dot, dashed (-..-) line indicates the subject had a decrease greater than the minimal difference. Dashed line $(---)=$ no change, dashed and dotted line (- . -) = decrease, and dotted line (...) = increase.

Figure 9. Individual responses for $\mathrm{VO}_{2}$ peak from PRE- to POST-test for the FEN supplement group. Solid line indicates the subject had an increase greater than the minimal difference, dashed, double dot, dashed (-..-) line indicates the subject had a decrease greater than the minimal difference. Dashed line $(---)=$ no change, dashed and dotted line (- . -) = decrease, and dotted line (...) = increase. 34

Figure 10. Individual responses for $\mathrm{VO}_{2}$ peakfrom PRE- to POST-test for the PLA supplement group. Solid line indicates the subject had an increase greater than the minimal difference, dashed, double dot, dashed (-...) line indicates the subject had a decrease greater than the minimal difference. Dashed line $(--)=$ no change, dashed and dotted line (- . -) = decrease, and dotted line (...) = increase.

Figure 11. Individual responses for time to exhaustion from PRE-to POST-test for the CUR supplement group. Solid line indicates the subject had an increase greater than the minimal difference, dashed, double dot, dashed (- . - -) line indicates the subject had a decrease greater than the minimal difference. Dashed line (---) = no change, dashed and dotted line (- . -) = decrease, and dotted line (...) = increase. 35

Figure 12. Individual responses for time to exhaustion from PRE-to POST-test for the FEN supplement group. Solid line indicates the subject had an increase greater than the minimal difference, dashed, double dot, dashed (- . . -) line indicates the subject had a decrease greater than the minimal difference. Dashed line (---) = no change, dashed and dotted line (- . -) = decrease, and dotted line (...) = increase. 36 
Figure 13. Individual responses for time to exhaustion from PRE-to POST-test for the PLA supplement group. Solid line indicates the subject had an increase greater than the minimal difference, dashed, double dot, dashed (- . .) line indicates the subject had a decrease greater than the minimal difference. Dashed line (---) = no change, dashed and dotted line (- . -) = decrease, and dotted line (...) = increase. 36 


\section{CHAPTER 1. INTRODUCTION}

Curcumin is an active ingredient of polyphenolic curcuminoids, which are found in the spice turmeric (Boonla et al. 2014). Curcumin has been well studied in clinical investigations over the last few decades and has been shown to have anti-inflammatory, anti-cancer, anti-diabetic, anti-hypertensive, anti-dementia and antioxidant properties (Boonla et al. 2014). Many of the purported effects of curcumin are related to reducing oxidative stress as well as improving markers of vascular function and cardiac performance in animal models (Boonla et al. 2014). Recent studies, however, have also demonstrated that curcumin supplementation may increase blood antioxidant capacity and reduce exercise-induced oxidative stress in humans (Takahashi et al. 2013). Specifically, curcumin supplementation has been shown to offset muscle damage and functional deficits associated with the eccentric nature of downhill running (Davis et al. 2007). Previous investigators have reported decreased markers of muscle damage, including inflammatory cytokines and creatine kinase after curcumin supplementation compared with a placebo (Davis et al. 2007). These decreases in inflammatory markers may have offset some of the performance deficits associated with eccentric exerciseinduced muscle damage (Davis et al. 2007).

Recently, curcumin has been shown to decease the accumulation of metabolic byproducts such as lactate and ammonia (Sahin et al. 2016) during acute exercise bouts. Specifically, it was reported (Sahin et al. 2016) that decreased levels of lactate and muscle malondialdehyde were associated with increased run times to exhaustion in rodents supplemented with curcumin compared to a placebo. It was suggested that the 
increased clearance of metabolic byproducts with curcumin supplementation might potentially be linked to increased production of nitric oxide (NO), a potent vasodilator, and the subsequent vasodilatory response (Boonla et al 2014). Thus, previous research (Boonla et al. 2014, Sahin et al. 2016, Takahashi et al. 2013) has shown the potential for curcumin to have a wide spectrum of bioactivities that may promote health as well as improve endurance performance. There are currently limited data, however, regarding the effects of curcumin supplementation on submaximal and maximal exercise performance in humans (Boonla et al. 2014).

The physical working capacity at the fatigue threshold $\left(\mathrm{PWC}_{\mathrm{FT}}\right)$ is an electromyographic (EMG) technique that theoretically estimates the highest power output that can be maintained for an extended period of time without evidence of neuromuscular fatigue (deVries et al 1987, 1990). Specifically, during a continuous incremental test, the $\mathrm{PWC}_{\mathrm{FT}}$ is defined as the average of the highest power output that results in no significant change in EMG amplitude (a nonsignificant slope coefficient) and the lowest power output that results in a significant increase in EMG amplitude (a significant positive slope coefficient) (deVries et al 1987, 1990). An important contributing factor to the etiology of neuromuscular fatigue is the accumulation of various metabolic byproducts and/or ions (i.e., inorganic phosphate, hydrogen ions, ammonia and potassium) (Camic et al 2010, Zuniga et al 2010, Bergstrom et al 2013) that interfere with skeletal muscle contractile mechanism and propagation of the action potential along the sarcolemma (Clausen 2013, Enoka and Stuart 1992, Gladden 2004, MacLaren et al 1989, Robergs et al 2004, Westerblad et al 2002). Previously, investigators (Camic et al. 2010) examined the effects of daily oral administration of arginine-based supplements for 4 weeks on the $\mathrm{PWC}_{\mathrm{FT}}$ in 
aerobically untrained men. Like curcumin, one of the physiological actions of arginine is to up regulate nitric oxide (NO) production and increase endothelium-dependent vasodilation (Boonla 2014). The authors (Camic et al. 2010) reported significant increases in the $\mathrm{PWC}$ FT after supplementation with arginine compared to a placebo. Thus, it is possible that curcumin supplementation may work similarly to arginine to delay the onset of neuromuscular fatigue and the $\mathrm{PWC}_{\mathrm{FT}}$ through $\mathrm{NO}$-mediated vasodilation (Boonla et al. 2014) and improved metabolic byproduct clearance (Sahin et al 2016).

Previous investigators (Sahin et al. 2016) have reported increased run times to exhaustion in rodents supplemented with curcumin compared to a placebo. Consistent with the findings by Sahin et al. (2016), Huang et al. (2015) reported that curcumin supplementation dose-dependently increased grip strength and endurance performance compared to the vehicle treatment (Huang et al 2015). Furthermore, previous investigators have shown that curcumin supplementation, in combination with endurance training, has the potential to accelerate mitochondrial biogenesis in skeletal muscle by increasing cAMP levels (Hamidie et al 2015). Peripheral cellular changes, such as increased mitochondrial density, have been shown to improve submaximal performance, (Basset 2000). Thus, it is possible the cellular effects of curcumin to improve markers of aerobic respiration may also delay time to fatigue and improve maximal oxygen consumption $\left(\mathrm{V}_{2}\right)$ during incremental exercise.

Curcumin has poor absorption into the intestines and consequently low bioavailability in the blood, and thus has been combined with other compounds to improve absorption and increased blood concentration levels (Krishnakumar et al 2012). One ingredient that has been studied in combination with curcumin is the naturally 
growing plant, fenugreek soluble fiber (Krishnakumar et al 2012). The galactomannan component of fenugreek has been combined with curcumin (CurQfen $\AA$ ) to increase absorption from the small intestine (Krishnakumar et al 2012). Galactomannan is a soluble fiber guar gum, and a potential active component of fenugreek seeds (Srichamroen et al 2008). Specifically, galactomannans from fenugreek have been shown to have increased effects on glycemic and lipidemic status in rats (Srichamroen et al 2008). Thus, it is possible the fenugreek component in CurQfen ${ }^{\circledR}$ may increase FFA release and decrease insulin levels, supporting improvements in submaximal exercise performance markers. No previous studies, however, have examined the effects of supplementation with curcumin in combination with fenugreek soluble fiber or fenugreek soluble fiber alone on submaximal or maximal indices of endurance performance. Therefore, the purpose of this study was to examine the effects of curcumin in combination with fenugreek (CUR) or fenugreek soluble fiber alone (FEN) on the neuromuscular fatigue threshold $\left(\mathrm{PWC}_{\mathrm{Fr}}\right)$, time to exhaustion $\left(\mathrm{T}_{\text {lim }}\right)$ on a graded exercise test (GXT), and $\dot{V} \mathrm{O}_{2}$ peak in untrained subjects. Based on the results of previous studies (Boonla et al. 2014, Sahin et al. 2016, Takahashi et al. 2013), it was hypothesized that curcumin supplementation would delay the onset of neuromuscular fatigue and increase the $\mathrm{PWC}_{\mathrm{FT}}$ as well as increase $\mathrm{T}_{\mathrm{lim}}$ and $\dot{V} \mathrm{O}_{2}$ peak compared to a placebo. 


\section{CHAPTER 2. REVIEW OF LITERATURE}

\subsection{Curcumin: Mechanisms of Action}

Boonla et al. (2014)

Previous studies have shown that increased production of oxidants, reduced nitric oxide (NO) bioavailability, and reduced activity of antioxidants in the vascular system are involved in hypertension and cardiovascular disease. Research has found that polyphenols play an important role in the maintenance of health and prevention of diseases. Curcumin is an active ingredient of polyphenolic curcuminoids extracted from the rhizomes of the spice turmeric. Curcumin is well known for its anti-inflammatory, anti-cancer, anti-diabetic, anti-hypertensive, anti-dementia and antioxidant properties and recent studies have shown that curcumin reduces oxidative stress in rodent models. However, there have been no reports about the activity of curcumin in 2 kidney-1clip (2K1C)-induced hypertension-induced endothelial dysfunction and vascular remodeling. Therefore, the present study was designed to investigate the effects of curcumin on $2 \mathrm{~K}$ 1C - induced hypertension, endothelial dysfunction, vascular remodeling and oxidative stress in male rats. The experimenters randomly placed the rats into the sham operated groups or $2 \mathrm{~K}-1 \mathrm{C}$ groups. Hypertension was induced by clipping the left renal artery with a silver clip in groups 3-5. Group 1 rats were sham operated and received the control, propylene glycol, and group 2 sham operated rats received $100 \mathrm{mg} / \mathrm{kg} /$ day of curcumin. Groups 3-5 were clipped rats that received propylene glycol alone, $50 \mathrm{mg} / \mathrm{kg} /$ day of curcumin and $100 \mathrm{mg} / \mathrm{kg} / \mathrm{day}$, respectively. After 6 weeks of treatment, the authors found that curcumin ameliorated hemodynamic performance in $2 \mathrm{~K}-1 \mathrm{C}$ hypertensive rats, by reducing blood pressure, increasing hindlimb blood flow and decreasing hindlimb 
vascular resistance. The results also showed that curcumin attenuated hypertensioninduced oxidative stress and vascular structural modifications. Overall, the authors concluded that the mechanisms responsible for the antihypertensive action of curcumin in 2K-1C hypertension-induced endothelial dysfunction and vascular remodeling involve the improvement of nitric oxide bioavailability and a reduction in oxidative stress.

Sahin et al. (2016)

The purpose of this study was to investigate the effects of curcumin supplementation on oxidative stress markers, exercise endurance, performance, and changes in serum and muscle proteins in rats following a test to exhaustion. This study used 28 male rats, assigned to one of four different treatment groups 1) control, 2) no exercise + curcumin supplement, 3) exercise only 4 ) exercise + curcumin supplementation. Supplementation was administered every day for 6 weeks in the form of $100 \mathrm{mg} \cdot \mathrm{kg}^{-1} \mathrm{CurcuW}$ in oral tablets. The rodents completed a 6 -week exercise period in which they exercised on a motor driven rodent treadmill 5 days a week for 45 minutes at a rate of $25 \mathrm{~m} \cdot \mathrm{min}^{-1}$. Following the 6-week exercise protocol, the rats were sacrificed and blood and muscle samples were analyzed for muscle markers, oxidative stress, and antioxidant markers. The results showed there were decreased levels of lactate and muscle malondialdehyde in the treatment groups which received the curcumin supplement. The run time to exhaustion also improved in the supplement groups and muscle nuclear factor and heat shock protein levels were significantly lower in group 4 (exercise + curcumin). The results of this study suggested that curcumin may have the ability to help prevent muscle damage during exercise by regulating certain proteins, which can lead to increased endurance and exercise performance. 
Takahashi et al. (2013)

The purpose of this study was to investigate the effects of curcumin supplementation on exercise-induced oxidative stress (reactive oxygen species [ROS]) in humans. Ten untrained male subjects (23-30 years of age) were included in this study. Prior to supplementation, the subjects performed a baseline test to determine the $\mathrm{VO}_{2}$ max. Each subject then participated in 3 laboratory- based trials in a randomized order: (1) placebo (control), (2) single curcumin supplementation (90 mg only before exercise) and (3) double curcumin supplementation (90 $\mathrm{mg}$ before and immediately after exercise). A washout period of at least one week was included between each condition trial. For each trial, the subjects exercised at $65 \%$ of their $\mathrm{VO}_{\text {2max }}$ for 60 minutes. Blood samples were taken pre-exercise, immediately after, and 2 hours post exercise. The results showed that the curcumin supplementation trials had decreased exercise-induced serum concentrations of d-ROMs and TRX-1 and increased serum BAP concentrations and plasma GSH after exercise. There were no changes in heart rate or RPE among the three trials. These findings suggested that curcumin supplementation may increase blood antioxidant capacity, and, therefore help to decrease exercise-induced oxidative stress.

\section{Summary}

Curcumin, an active ingredient of polyphenolic curcuminoids extracted from the rhizomes of the spice turmeric is well known for its an anti-inflammatory, anti-cancer, anti-diabetic, anti-hypertensive, anti-dementia and antioxidant properties (Boonla et al 2014). Recently, curcumin has also been shown to decrease hypertension-induced oxidative stress and vascular structural modifications (Boonla et al. 2014). Curcumin has also shown to reduce blood pressure, increase hindlimb blood flow, and decreasing 
hindlimb vascular resistance in mice. In addition, curcumin supplementation has been shown to improve NO bioavailability and reduce oxidative stress (Boonla et al. 2014), A study by Takahashi et al. (2013) showed that curcumin also has physiological effects on human subjects as well, including decreased exercise-induced oxidative stress. These findings extended those of the rodent study by Boonla et al. (2014) and suggested curcumin may have the potential to improve endurance performance in humans (Takahashi et al. 2013).

\subsection{Physiological and Performance Effects of Curcumin supplementation}

Davis et al. (2007)

The purpose of this study was to investigate the effects of curcumin supplementation on muscle damage and functional deficits associated with downhill running in rodents. The heavy eccentric muscle contraction in downhill running has been linked to increased muscle fiber damage, inflammation, and delayed onset muscle soreness (DOMs) as well as several other functional deficits. The mice used in this study were randomly assigned to one of four groups, 1) downhill running + placebo, 2) downhill running + curcumin, 3) uphill running + placebo, or 4) uphill running + curcumin. The "control" in this study was the uphill running group, since it has been shown to produce little to no muscle damage and inflammation. Previous studies have shown that voluntary and involuntary running in rodents was significantly decreased following a downhill run for up to 4 days. The suggested reasoning behind this decrease in performance is due to inflammation including increases in brain IL-1 associated with downhill running. The authors hypothesized that the anti-inflammatory activity of 
curcumin would decrease recovery time following a downhill run and increase running performance due to a reduction in inflammation-induced deficits in regeneration of muscle fibers, soreness, and central nervous system fatigue. Curcumin supplementation was administered to the mice in the form of a food pellet containing $10 \mathrm{mg}$ of curcumin powder (Down-Cur and Up-Cur), and the placebo groups were fed a food pellet without curcumin (Down-Plac and Up-Plac)] every day for 3 days prior to the up/downhill run. Following supplementation, the mice performed their initial uphil/downhill run tests. Two groups of mice (Down-Plac and Down- Cur) ran on a rodent treadmill at $-14 \%$ grade and the other two groups (Up- Plac and Up- Cur) ran at a $+14 \%$ grade at a rate of $22 \mathrm{~m} / \mathrm{min}$ for 150 minutes. Following the uphil//downhill runs, a subset of mice were used to assess recovery of running performance. These mice ran to fatigue on a motorized treadmill at $36 \mathrm{~m} / \mathrm{min}$ and $8 \%$ grade at either 48 or $72 \mathrm{~h}$ post-up/downhill run. A second subset of mice were used to assess recovery of voluntary activity following the up/downhill run on the rodent treadmill. Voluntary wheel running activity during the "active" dark period was measured automatically for 7 consecutive days. The results showed that downhill running was in fact associated with an increase in inflammatory cytokines and creatine kinase, however, the increase in these metabolic byproducts were offset in the group that received the curcumin supplement. The groups who received the curcumin supplement also showed increased voluntary run times as compared to those who received the placebo and the groups who participated in the involuntary run. The importance of these results suggest that the anti-inflammatory phytochemical curcumin can decrease recovery time following high intensity eccentric exercise, known to produce muscle damage and DOMS. Curcumin also appears to be capable of enhancing a 
behavioral response (spontaneous running), which implied an important central nervous system component (e.g., drive, motivation) that may be related to less pain and reduced inflammatory cytokines.

Huang et al. (2015)

The aim of this study was to evaluate the potential benefits of curcumin supplementation in a mouse model on physical performance test and exhaustive swimming. The authors hypothesized that curcumin supplementation may mediate exercise-induced metabolites, energy distribution, and even improve physical performance. This experiment used male mice, which were randomly placed into one of four groups to receive treatment by oral gavage: placebo control (water), curcumin supplement at $12.3 \mathrm{~mL} \cdot \mathrm{kg}^{-1 \cdot} \mathrm{day}^{-1}, 24.6 \mathrm{~mL} \cdot \mathrm{kg}^{-1 \cdot} \mathrm{day}^{-1}$, and $61.5 \mathrm{~mL} \cdot \mathrm{kg}^{-1 \cdot} \mathrm{day}^{-1}$. The supplementation period lasted a total of 28 days. Each mouse subject was tested for its forelimb grip strength, and completed an exhaustive swimming test to evaluate performance and time to fatigue. The physical fatigue-associated biomarkers included, serum lactate, ammonia, blood urea nitrogen (BUN), and glucose and tissue damage markers such as aspartate transaminase (AST), alanine transaminase (ALT), and creatine kinase (CK). The results of this study showed a dose-dependent response for curcumin supplementation on increased grip strength and endurance performance and significantly decreased lactate, ammonia, BUN, AST, ALT, and CK levels post exercise. Muscular glycogen content, an important energy source for exercise, was also significantly increased in the groups who received the curcumin supplement as compared to the placebo. From these results, the authors concluded that curcumin supplementation may 
have a wide spectrum of bioactivities for promoting health, improving exercise performance, and preventing fatigue.

Summary:

Recent studies have observed the physiological and performance effects of curcumin supplementation, particularly in animal subjects. Davis et al. (2007) reported increased voluntary run times for those mice receiving the curcumin supplement compared to those who received the placebo. These results suggested that the antiinflammatory phytochemical curcumin can decrease recovery time following high intensity eccentric exercise, known to produce the most muscle damage and DOMS. Interestingly, due to the increased voluntary run times, curcumin appears to be capable of enhancing a behavioral response, which implies an important central nervous system component (e.g., drive, motivation) that may be related to less pain and perhaps reduced inflammatory cytokines (Davis et al. 2007). Curcumin supplementation also has an effect on lactate, ammonia, BUN, AST, ALT, and CK (physical fatigue-associated biomarkers) levels post exercise. Huang et al. (2015) found a significant decrease in the levels of these metaoblites (lactate, ammonia, etc.) post exercise in the subjects given curcumin supplementation compared with a placebo. The authors (Huang et al. 2015) also reported increased grip strength and endurance performance among the supplemented groups. Muscular glycogen content, an important energy source for exercise, was also significantly increased in the groups who received the curcumin supplement as compared to the placebo (Huang et al. 2015). From the results of these studies (Davis et al. 2007, Huang et al. 2015), it appears that curcumin supplementation may have a wide spectrum 
of bioactivities for promoting health, improving exercise performance, preventing fatigue, and even a potential CNS component.

\subsection{Timing and Dosage of Curcumin Supplementation}

Krishnakumar et al. (2012)

Curcumin has been shown to have many positive physiological actions and has shown not to be toxic even at very high doses of 8-12 g/day, however, despite the safety, efficacy, and well-tracked mechanisms of action, curcumin has poor systematic bioavailability upon oral administration due to inadequate intestinal absorption, rapid metabolism and short half-life. The low bioavailability of curcumin limits its use as a therapeutic agent, therefore the purpose of this study was to investigate the bioavailability of curcumin combined with the spice fenugreek as a vehicle for administration in animals and humans. 8 human male subjects participated in this study. Each subject was tested with the unformulated and formulated curcumin supplements. For the first test, each subject was first given two capsules of $500 \mathrm{mg}$ of unformulated curcumin and $2 \mathrm{ml}$ blood sample were withdrawn at $0,0.5,1,3,5,8$ and $24 \mathrm{~h}$ post-dose for analysis. After 1 week of curcumin feeding, the subjects were given three capsules of $500 \mathrm{mg}$ containing newly formulated curcumin supplement (containing curcumin and fenugreek) and the same protocol was repeated (the dosage of the three formulated capsules was equivalent to 600 $\mathrm{mg}$ of curcumin). The same was repeated again after 1 week with $250 \mathrm{mg}$ formulated supplement and the blood samples were withdrawn for analysis (the $250 \mathrm{mg}$ dosage was equivalent to $100 \mathrm{mg}$ of unformulated curcumin). The results of this study found that in humans, the absorption of curcumin + fenugreek supplement was 15.8 times higher in 
oral dosages of $1500 \mathrm{mg}$ (equivalent to $600 \mathrm{mg}$ curcumin), 12.9 times higher at $250 \mathrm{mg}$ (equivalent to $100 \mathrm{mg}$ curcumin) of CGM as compared to $1000 \mathrm{mg}$ of unformulated curcumin. These results indicate that the bioavailability of curcumin is not only increased by combining it with fenugreek, but is also dose dependent.

\section{Summary:}

The observed physiologic effects of curcumin and its use as a therapeutic agent have been limited due to its poor systematic bioavailability upon oral administration which is related to its inadequate intestinal absorption, rapid metabolism, and short halflife. Krishnakumar et. al. (2012) investigated the bioavailability of curcumin combined with the herb fenugreek in animals and humans. In the human subjects who received the formulated curcumin supplement, absorption was 15.8 times higher in oral dosages of $1500 \mathrm{mg}$ (equivalent to $600 \mathrm{mg}$ curcumin), and 12.9 times higher at $250 \mathrm{mg}$ (equivalent to $100 \mathrm{mg}$ curcumin) as compared to $1000 \mathrm{mg}$ of unformulated curcumin. The results of this study indicate that the bioavailability of curcumin is dose dependent and increased by combining it with fenugreek.

\subsection{Development of the Physical Working Capacity at the Fatigue Threshold Model} deVries et al. (1982)

This study examined the relationship between two fatigue thresholds (critical power $[\mathrm{CP}]$ and fatigue threshold $[\mathrm{FT}])$, derived from unique methodologies. The $\mathrm{CP}$ was determined from four, exhaustive, constant power output cycle ergometer workbouts. The $\mathrm{CP}$ was defined as the slop of the relationship between the total work performed and the time to exhaustion for the four workbouts. In addition, the anaerobic threshold (AT) was 
determined from gas exchange and ventilatory parameters measured during an incremental test to exhaustion. The FT was then estimated by measuring integrated electromyographic amplitude (IEMG) from the quadriceps femoris muscle during four, constant power output cycle ergometer workbouts. This study utilized a unipolar lead system, with surface electrodes placed on the lateral portion of the dominant quadriceps femoris muscle and reference electrode over the anterior superior iliac spine (ASIS). The IEMG data was plotted over 10 second periods as a function of time during three or four workbouts of various power outputs on the cycle ergometer. The slope coefficients from each power output were determined and then power output was plotted against the slope coefficients for each of three or four workbouts (fatigue slope coefficient on the $\mathrm{x}$ axis and power output in W on the y). The FT was identified as the point of zero slope, estimated from the Y intercept value and expressed in W. The FT was defined as the highest constant power output that could be maintained during cycle ergometry without an increase in IEMG activity. This study demonstrated a linear relationship between time and increased IEMG activity $\left(\mathrm{r}^{2}=0.88-0.99\right)$. The FT ranged from $124-284 \mathrm{~W}$, with a mean value of $190.5 \pm 14.0 \mathrm{~W}$. In addition, the $\mathrm{CP}$ and $\mathrm{FT}$ as well as the AT and FT were compared. They found that the relationship between AT and FT $(r=0.903, p<0.001)$ was stronger than that between FT and $\mathrm{CP}(\mathrm{r}=0.860, \mathrm{p}<0.001)$ and there was no significance between AT and FT, CP was less than FT. The authors concluded that due to the correlation found among the three variables, $\mathrm{CP}, \mathrm{AT}$ and FT there may be a single phenomenon that underlies them all, and IEMG estimation of FT may provide an alternative to the measurement of AT. 
deVries et al. (1987)

The purpose of this study was to develop a test utilizing electromyographic (EMG) responses from submaximal discontinuous work bouts on the bicycle ergometer to determine the physical working capacity at the fatigue threshold $\left(\mathrm{PWC}_{\mathrm{FT}}\right)$. The subjects for this study included 32 males, 18-29 years old, with fitness levels ranging from highly trained to untrained and sedentary. The same IEMG equipment and procedures were used in this study as in the previous study conducted by deVries et al. (1982). The onset of blood lactate accumulation (OBLA), percent heart rate range (\%HRR) at the $\mathrm{PWC}_{\mathrm{FT}}$, heart rate- workload (HR-WL) relation, critical power (CP) and ratings of perceived exertion (RPE) at the $\mathrm{PWC}_{\mathrm{FT}}$ were measured for each subject. These measurements were used to examine the validity of the $\mathrm{PWC}_{\mathrm{FT}}$ model through correlational and regression analyses. The procedures for measuring the $\mathrm{PWC}_{\mathrm{FT}}$ included multiple discontinuous work bouts on the bicycle ergometer at increasing intensities, with the measurement of IEMG amplitude. The subject's heart rate returned to within 10 $\mathrm{b} \cdot \mathrm{min}^{-1}$ of the resting value before the next work bout was initiated. The first work bout was performed at $420 \mathrm{kpm} \cdot \mathrm{min}^{-1}$ and increased by $420 \mathrm{kpm} \cdot \mathrm{min}^{-1}$ for each subsequent work bout ( 3 total). The $\mathrm{PWC}_{\mathrm{FT}}$ was determined as the average of the highest power output with no change in IEMG amplitude over time and the lowest power output that resulted in an increase in IEMG amplitude. Seventeen of the subjects were retested on a different day to determine the reliability of the $\mathrm{PWC}_{\mathrm{Fr}}$ test. The authors found that $\mathrm{PWC}_{\mathrm{Fr}}$ was highly reproducible, with a test-retest correlation of $r=0.947$ and a SEE of 145.6 $\mathrm{kpm} \cdot \mathrm{min}^{-1}(9.7 \%$ of the mean PWC $\mathrm{FT})$. The results also showed the OBLA, \% HRR, and 
HR-WL relationship accounted for $70 \%$ of the total variance in the $\mathrm{PWC}_{\mathrm{FT}}$. In conclusion, the $\mathrm{PWC}_{\mathrm{FT}}$ model was shown to be valid and highly reproducible, providing an attractive alternative to supramaximal tests for untrained or sedentary individuals.

deVries et al. (1990)

The purpose of this study was to examine alternative methodological procedures for the determination of the physical working capacity at the fatigue threshold $\left(\mathrm{PWC}_{\mathrm{FT}}\right)$ to improve the practical application and extend the utility of the test. This study examined the use of a continuous test protocol rather than a discontinuous protocol. In addition, the $\mathrm{PWC}_{\mathrm{Fr}}$ estimates were compared for treadmill and bicycle ergometer exercise, from a bipolar electromyographic (EMG) lead system and a unipolar lead system, as well as the potential for residual fatigue from tests repeated 24 hours apart. Twenty subjects participated in this study, with a wide age (19-70 years) range and different fitness levels. Each subject participated in one or more of the experiments conducted. For each experiment EMG data and fatigue curves were recorded as previously described by deVries et al. (1987). The initial power output was set based on the subject's estimate of their fitness level and abilities and was then incrementally increased by $12-25 \mathrm{~W}$ at each stage for the bicycle ergometer tests and by one to two percent grade increases for the treadmill test. The first experiment was a comparison continuous versus discontinuous protocol on the bicycle ergometer. For the discontinuous test, the subjects rested until their HR was within $10 \mathrm{~b} \cdot \mathrm{min}^{-1}$ of their resting value between each power output or grade increase. The continuous test followed the same procedures as the discontinuous test except for there was no rest interval between each increase in intensity. The PWCFT was determined as the average of the highest power output with no change in IEMG 
amplitude over time and the lowest power output that resulted in an increase in IEMG amplitude. There was no mean difference between the $\mathrm{PWC}_{\mathrm{FT}}$ estimates from the discontinuous versus continuous test on the bicycle ergometer and they were highly correlated $(\mathrm{r}=0.856)$. The PWCFT estimates from the treadmill versus bicycle ergometer involved 6 of the 20 subjects, for both the continuous and discontinuous protocols. Since there is no direct measurement of work or power for exercise on a treadmill, to determine

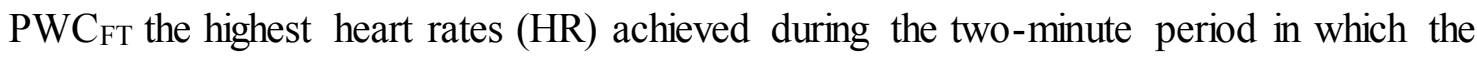
fatigue threshold was reached (i.e., increase in EMG amplitude) were compared from the bicycle ergometer test and treadmill test. The EMG data were collected from the quadriceps and gastrocnemius muscles for the treadmill test. The results showed that there was no significant difference between the mean $\mathrm{HR}$ at the $\mathrm{PWC}_{\mathrm{FT}}$ between the two different muscle groups, however, the HR at the $\mathrm{PWC}_{\mathrm{FT}}$ was $20-35 \mathrm{~b} \cdot \mathrm{min}^{-1}$ lower on the bicycle ergometer compared to the treadmill. The unipolar versus bipolar lead systems test showed that the mean voltages from the unipolar measurements were significantly greater than for the bipolar, but they were well correlated $(\mathrm{r}=0.841)$. The $\mathrm{PWC}_{\mathrm{FT}}$ estimates from unipolar and bipolar were not significantly different $(t=1.09 \mathrm{p}>0.05)$. Furthermore, the effect of 24-hour repeated tests showed that there was no residual fatigue effect for discontinuous $\mathrm{PWC}_{\mathrm{FT}}$ tests repeated at a 24 hour interval.

\section{Summary}

The Physical Working Capacity at the Fatigue Threshold Model $\left(\mathrm{PWC}_{\mathrm{FT}}\right)$ was developed by deVries et al. (1982) to examine the onset of neuromuscular fatigue. This model utilized surface electromyography (EMG) from the quadriceps muscle. The amplitude (AMP) of the EMG signal was plotted versus time for a series of constant 
work rate rides. The $\mathrm{PWC}_{\text {гт }}$ was defined as the highest constant power output that could be maintained during cycle ergometry without an increase in EMG AMP. deVries et al. expanded upon the initial study, and in 1990 an experiment to determine factors that may affect the estimation of the $\mathrm{PWC}_{\text {ғг }}$ Overall, it was concluded that the model could be extended to treadmill running, conducted using bipolar or unipolar leads, but most importantly, that a continuous exercise protocol could be implemented instead of the previously utilized, discontinuous protocol.

\subsection{Utilization of $\mathrm{PWC}_{\mathrm{rr}}$ to Examine the Efficacy of Nutritional Supplements to Improve Performance}

Camic et al (2010a)

The purpose of this study was to examine the effects of 4 weeks of arginine-based supplementation on the physical working capacity on the fatigue threshold (PWCFT). Arginine serves as a precursor in the production of nitric oxide (NO) and plays an important role in endothelium-dependent vasodilation. Due to these physiological effects, arginine supplementation may help to increase blood flow to the myocardium and skeletal muscle. This was a double-blind, placebo-controlled, parallel design which included 50 college aged men who were randomly placed into one of three groups: 1) placebo, 2) $1.5 \mathrm{~g}$ arginine or 3) $3.0 \mathrm{~g}$ arginine. All of the subjects performed a presupplementation (PRE) incremental test to exhaustion on a cycle ergometer to determine the PWCFT and performed the exact same protocol after 4 weeks of arginine supplementation. The subjects were instructed to take one dose (4 pills) of their assigned supplement on an empty stomach immediately after they woke up every morning with 16 
oz. of water for 28 days. The subjects were instructed not to change their dietary or exercise habits during the supplementation period. At their post supplementation (POST) visit subjects took one dose 60 minutes prior to the test. The incremental test to exhaustion protocol has previously been described by deVries et. al. (1987) Surface EMG signals were recorded from the vastus lateralis using a bipolar electrode arrangement during the incremental tests for the determination of the PRE and POST supplementation PWCFT values. The results of the study indicated significant increases in the PWCFT values for the $1.5 \mathrm{~g}(22.4 \%)$ and $3.0 \mathrm{~g}(18.8 \%)$ supplement groups, but no change for the placebo group (-1.6\%). These findings were likely due to the physiologic actions of arginine combined with grape seed extract, which help reduce the concentrations of metabolic by-products produced during exercise, improved blood flow associated with increased NO synthesis and decreased endothelin production. In conclusion, arginine based supplements can be used as an ergogenic aid for untrained individuals to increase time to fatigue and potentially improve exercise performance.

\section{Summary}

Arginine has been shown to serve as a precursor in the production of nitric oxide (NO) and plays an important role in endothelium-dependent vasodilation (Camic et al. 2010a). Due to these physiological effects, arginine supplementation may help to increase blood flow to the myocardium and skeletal muscle and may have an affect on the $\mathrm{PWC}_{\mathrm{Fr}}$. Camic et al. (2010a) conducted a study in which examined the effects 28 days of supplementation with a placebo, $1.5 \mathrm{~g}$ arginine, and $3.0 \mathrm{~g}$ arginine on the $\mathrm{PWC}_{\mathrm{FT}}$. The results of the study (Camic et al. 2010a) indicated significant increases in the PWC $\mathrm{FT}_{\text {for }}$ the $1.5 \mathrm{~g}(22.4 \%)$ and $3.0 \mathrm{~g}(18.8 \%)$ supplement groups, but no change for the placebo 
group $(-1.6 \%)$. These findings were likely due to the physiologic actions of arginine combined with grape seed extract, which help reduce the concentrations of metabolic byproducts produced during exercise, improved blood flow associated with increased NO synthesis, and decreased endothelin production. In conclusion, supplementation with a compound (arginine) that improves endothelial function and blood flow can be used as an ergogenic aid for untrained individuals to increase time to fatigue and potentially improve exercise performance. 


\section{CHAPTER 3. METHODS}

\subsection{Experimental Design and Supplementation}

The present study used a randomized, double-blind, placebo- controlled, parallel design. For this study, there were three groups, two experimental groups and one control. Due to the low bioavailability of curcumin, it was taken in combination with galactomannan soluble dietary fiber from fenugreek seeds in a supplement called CurQfen ${ }^{\circledR}$, which has been shown to significantly increase plasma concentrations of curcumin (Krishnakumar et al. 2012). The first visit to the laboratory was to determine each subject's $\mathrm{PWC}_{\mathrm{FT}} \mathrm{PRE}$,

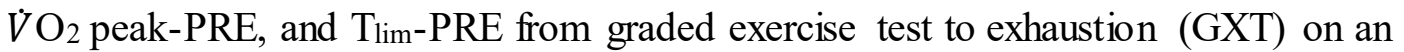
electronically braked ergometer. Following the PRE-test, 47 subjects were randomly assigned to either the control group (placebo: PLA, n=15), CurQfen ${ }^{\circledR}$ (CUR, n=18), or fenugreek soluble fiber $(\mathrm{FEN})(\mathrm{n}=14)$ and were given instructions regarding the supplementation process. The supplements were taken daily for 28 days, in the morning before eating, with $16 \mathrm{oz}$. of water. The CurQfen ${ }^{\circledR}$ dosage was $500 \mathrm{mg}$ with $100 \mathrm{~g}$ of CurQfen $®$ powder that contained 37 to $38 \mathrm{~g}$ of natural curcuminoids (including curcumin (31g), demethoxy curcumin (6g) and bisdemethoxy curcumin (1.1g)) along with $60 \mathrm{~g}$ of de-bitterised fenugreek dietary fiber rich in galactomannans, and a 2 to $4 \%$ moisture content. The fenugreek only group was included to account for any extraneous effects of fenugreek soluble fiber and contained 300mg. During the supplementation period, the subjects were instructed not to change their dietary habits or increase their activity level. Food logs were kept for three days prior to the PRE- and POST-test to determine the total grams consumed for each macronutrient as well as total kilocalories. In total, 41 of the 47 subjects completed and returned the food logs for PRE- and POST-test and were included 
in the subsequent analyses of total kilocalories and grams of macronutrients consumed. The subjects checked in every week to ensure adherence to the protocol and compliance to the supplementation regimen. To be included in the final analyses, subjects had to demonstrate $>80 \%$ compliance (compliance $=[\#$ of capsules consumed / \# of capsules provided] x 100). After 28 days of supplementation, the subjects returned to the laboratory for their POST-test to determine $\mathrm{PWC}_{\mathrm{FT}}-\mathrm{POST}, \dot{V} \mathrm{O}_{2}$ peak-POST, and $\mathrm{T}_{\text {lim- }}$ POST.

\subsection{Subjects}

The subjects recruited for the present investigation were college-aged, aerobically untrained individuals. The subjects were not highly trained, specifically in bicycling, and participated in no more than 3 days a week of regular activity ( $<4$ hours per week). In total, 67 subjects were recruited to participate in this study, and 47 were included in the final analyses. Of the initial 67 subjects, three subjects withdrew from the study due to scheduling conflicts, one withdrew due to illness, and five were excluded due to equipment malfunctions. From the remaining 58 subjects, two were excluded due to an inability to complete minimal stage requirements for the GXT, and nine subjects did not have a detectable PWC $\mathrm{PWT}_{\text {at }}$ aither the PRE- or POST-test. All of the 47 subjects included in the final analyses were $>80 \%$ complaint (mean \pm SD: $27.88 \pm 0.51$ ). All of the subjects completed a health history questionnaire and met the following criteria: (a) no history of medical or surgical events that could significantly affect experimental results or increase the subjects risk of injury, these included cardiovascular disease, metabolic, renal, hepatic, or musculoskeletal disorders; (b) were not taking any medication that 
could significantly affect experimental results; (c) not currently using any nutritional supplements that could significantly affect experimental results; and (d) not presently participating in another clinical trial or ingestion of another investigational product. The study was approved by the University’s Institutional Review Board for Human Subjects, and all subjects were given a health screening and signed a written informed consent document before testing.

\subsection{Determination of $\dot{\mathrm{V}} 2$ peak}

Each subject performed a GXT to exhaustion on a calibrated electronically-braked cycle ergometer (Lode Corival, Groningen, The Netherlands) to determine the $\mathrm{PWC}_{\mathrm{FT}}, \dot{\mathrm{VO}}_{2}$ peak, and $\mathrm{T}_{\text {lim }}$ at PRE- and POST-test. The ergometer seat height was adjusted so that the subject's legs reach near full extension at the bottom of the pedal revolution. Toe clips were used to maintain pedal contact throughout the test and all subjects were equipped with a nose clip and a 2-way valve mouth piece to collect all expired air. Expired gas samples were collected and analyzed using a calibrated metabolic cart (TrueMax 2400, ParvoMedics, Sandy, UT). The gas analyzers were calibrated with room air and gases of known concentration prior to all testing sessions. In addition, subjects wore a Polar Heart Rate Monitor (Polar Electro Inc., Lake Success, NY), synchronized with the metabolic cart to obtain heart rate measures throughout the ride. The test began at $50 \mathrm{~W}$ at a cadence of $70 \mathrm{rev} \cdot \mathrm{min}^{-1}$ and the power output increased by $30 \mathrm{~W}$ every 2 min until voluntary exhaustion or the subject's pedal rate dropped below $70 \mathrm{rev} \cdot \mathrm{min}^{-1}$ for more than 10 seconds, despite strong verbal encouragement. The $\dot{\mathrm{VO}}_{2}$ peak was defined as the highest $\dot{\mathrm{VO}}_{2}$ value in the last 30 seconds of the test that met two of the following three 
criteria: 1) $90 \%$ of age-predicted heart rate; 2) respiratory exchange rate $>1.1$; and 3) a plateau of oxygen uptake (less than $150 \mathrm{~mL} \cdot \mathrm{min}^{-1}$ in $\mathrm{VO}_{2}$ over the last 30 seconds of the test).

\subsection{Electromyographic Measurements}

The EMG signals were measured from the vastus lateralis (VL) on the dominant leg during the incremental test. Prior to electrode placement, the skin at each site was shaved, carefully abraded, and cleaned with alcohol. A bipolar surface electrode (circular 24 mm, Kendall disposable EMG electrodes, Covidien LTD; Gosport Hampshire, UK) arrangement $(2.0 \mathrm{~cm}$ center-to-center) was placed based on the recommendations from the SENIAM Project for EMG electrodes placement (Hermans et al. 1999). Specifically, a reference line was drawn over the $\mathrm{VL}$, one-third of the distance between the lateral superior border of the patella and the anterior superior iliac spine. In addition, the electrode-placement site was located $5 \mathrm{~cm}$ lateral to the reference line so that the electrodes were over the VL muscle (Malek et al. 2006). A goniometer (Smith \& Nephew Rolyan, Inc., Menomonee Falls, WI) was used to orient the EMG electrodes at a $20^{\circ}$ angle to the reference line to approximate the pennation angle of the muscle fibers for the VL (Abe et al. 2000). The EMG signal was amplified (gain: $\times 1,000$ ) using differential amplifiers (EMG 150 BIOPAC Systems, Inc., Santa Barbra, CA, bandwidth $=10-500$ $\mathrm{Hz})$.

\subsubsection{Signal Processing}

The raw EMG signals were digitized at $1,000 \mathrm{~Hz}$, stored in a personal computer (MacBook Pro OSX, version 10.6.8, Apple Inc., Cuperino, CA) for subsequent analysis 
and processed with a custom program written with LabVIEW programming software (version 7.1, National Instruments, Austin, TX). The EMG signals will be bandpassfiltered (zero phase shift, fourth-order Butterworth) at 10-500 Hz. Continuous $10 \mathrm{~s}$ epochs for the EMG AMP (microvolts root mean square, $\mu$ Vrms) and EMG MPF (MPF in $\mathrm{Hz}$ ) were calculated. For the MPF analyses, each data segment was processed with a Hamming window and a discrete Fourier transform (DFT) algorithm in accordance with the recommendations of Hermens et al. (1999). The MPF were selected to represent the power spectrum on the basis of the recommendations of Hermans et al. (1999) and was calculated as described by Kwatny et al. (1970).

\subsection{Determination of the Physical Working Capacity at the Fatigue Threshold} $(\mathrm{PWC} F \mathrm{FT})$

The protocol used to determine the $\mathrm{PWC}_{\mathrm{FT}}$ has previously been described in detail by deVries et al. (1987) and was conducted simultaneously with the GXT. Briefly, the test began at a power output of $50 \mathrm{~W}$, and was increased by $30 \mathrm{~W}$ every 2 min until voluntary exhaustion or the subject could no longer maintain a pedal cadence of $70 \mathrm{rpm}$. During each 2-minute power output, 12, 10-second EMG samples were recorded from the VL muscle. The EMG amplitude values for each of the 12,10-second periods were plotted across time for each power output of the test. The PWC $\mathrm{FT}_{\mathrm{FT}}$ was defined as the average of the highest power output that results in a non-significant slope coefficient and the lowest power output that results in a significant positive slope coefficient. 


\subsection{Statistical Analyses}

Separate, one-way ANOVAs were used to determine if there were any significant differences among the PLA, FEN, and CUR groups for age, height, weight, $\mathrm{PWC}_{\mathrm{FT}}, \mathrm{V}_{2}$ peak, and Tlim, at PRE-test. The test-retest reliability (PRE- and POST-test values) for each dependent variable was determined using the $\mathrm{ICC}_{2,1}$ model (Weir, 2005) for the 15

subjects in the PLA group. The standard error of the measurement $(\mathrm{SEM}=\mathrm{SD} \sqrt{1-I C C})$ and minimal difference need to be considered real (MD) (MD $=S E M \times 1.96 \times \sqrt{2})$ were also calculated for the PLA group for each dependent variable. In addition, three separate paired samples t-test were used to determine if there were any significant changes in the $\mathrm{PWC}_{\mathrm{FT}}, \dot{V} \mathrm{O}_{2}$ peak, or $\mathrm{T}_{\text {lim }}$ from PRE- to POST-test for the PLA. Three separate, one-way ANCOVAs were used to examine differences among group for each dependent variable, $\mathrm{PWC}_{\mathrm{FT}}, \dot{V} \mathrm{O}_{2}$, and $\mathrm{T}_{\text {lim. The respective }}$ PRE-test value was used as the covariate. The total caloric (kilocalories) and macronutrient (grams of carbohydrate, fat, and protein) intake was analyzed with separate, 3 (Group: CUR, FEN, and PLA) x 2 (Time: PRE and POST) mixed-factorial ANOVAs. A significant main effect was followed up with one-way ANOVAs and independent samples $\mathrm{t}$-test. An alpha of $\mathrm{p} \leq 0.05$ was considered statistically significant for all comparisons.

\section{CHAPTER 4. RESULTS}

The results of the one- way ANOVAs comparing pre-test values indicated that there were no significant mean group differences for the $\mathrm{PWC}_{\mathrm{FT}}(\mathrm{F}=0.705, \mathrm{p}=0.500), \dot{V} \mathrm{O}_{2}(\mathrm{~F}=$ 1.367, $\mathrm{p}=0.266)$, or $\mathrm{T}_{\lim }(\mathrm{F}=0.988, \mathrm{p}=0.381)$ determined from the $\mathrm{GXT}$ or for age $(\mathrm{F}=$ $1.469 \mathrm{p}=0.241)$, height $(\mathrm{F}=0.050 \mathrm{p}=0.951)$, or weight $(\mathrm{F}=1.62 \mathrm{p}=0.209)$ values. 
Table 1. Demographic information and PRE test values for time to exhaustion $\left(\mathrm{T}_{\mathrm{lim}}\right), \dot{\mathrm{VO}}_{2}$ peak, and the physical working capacity at the fatigue threshold $\left(\mathrm{PWC}_{\mathrm{FT}}\right)$.

\begin{tabular}{|c|c|c|c|}
\hline Variable & Placebo $(n=15)$ & Fenugreek $(n=14)$ & Curcumin $(\mathrm{n}=18)$ \\
\hline Age (years) & $20.27 \pm 1.44$ & $20.86 \pm 1.56$ & $21.78 \pm 3.66$ \\
\hline Height $(\mathrm{cm})$ & $174.81 \pm 6.77$ & $175.07 \pm 7.58$ & $175.62 \pm 8.09$ \\
\hline Weight $(\mathrm{kg})$ & $73.24 \pm 12.83$ & $79.91 \pm 12.14$ & $71.90 \pm 13.89$ \\
\hline $\begin{array}{l}\text { Time to exhaustion } \\
\text { (min) }\end{array}$ & $13.87 \pm 2.69$ & $15.13 \pm 2.53$ & $15.25 \pm 3.60$ \\
\hline $\begin{array}{c}\dot{V} \mathrm{O}_{2} \text { peak }\left(\mathrm{ml} \cdot \mathrm{kg}^{-1} \cdot \mathrm{min}^{-}\right. \\
\left.{ }^{-}\right)\end{array}$ & $39.20 \pm 8.95$ & $37.82 \pm 6.35$ & $42.32 \pm 8.22$ \\
\hline $\operatorname{PWC}_{\mathrm{FT}}(\mathrm{W})$ & $153.00 \pm 54.93$ & $176.43 \pm 43.12$ & $170.00 \pm 63.64$ \\
\hline
\end{tabular}

\subsection{Reliability Analysis}

The paired samples $\mathrm{t}$-tests indicated that there were no significant mean differences between PRE- and POST-test for the PLA group for the PWCFT $(t=-0.764 p=$ $0.458), \dot{V} \mathrm{O}_{2}$ peak $(\mathrm{t}=0.452 \mathrm{p}=0.658)$, and $\mathrm{T}_{\lim }(\mathrm{t}=-0.137 \mathrm{p}=0.893)$. The $\mathrm{ICC}_{2,1}$ values for the $\mathrm{PWC}_{\mathrm{FT}}, \dot{V} \mathrm{O}_{2}$ peak, and $\mathrm{T}_{\text {lim }}$ were $0.834,0.944$, and 0.953 , respectively. The SEM and MD values for the $\mathrm{PWC}_{\mathrm{FT}}, \dot{V} \mathrm{O}_{2}$ peak, and $\mathrm{T}_{\text {lim }}$ are presented in Table 2. 
Table 2. Results of the reliability analyses for the placebo group using PRE- and POSTtest values for the physical working capacity at the fatigue threshold $\left(\mathrm{PWC}_{\mathrm{FT}}\right), \dot{V} \mathrm{O}_{2}$ peak, and time to exhaustion $\left(\mathrm{T}_{\mathrm{lim}}\right)$.

\begin{tabular}{|c|c|c|c|c|c|c|}
\hline Subject & $\begin{array}{c}\text { PRE- } \\
\text { PWCFT } \\
(\mathrm{W})\end{array}$ & $\begin{array}{c}\text { POST- } \\
\text { PWCFT } \\
(\mathrm{W})\end{array}$ & $\begin{array}{c}\text { PRE- } \\
\dot{V} \mathrm{O}_{2} \text { peak } \\
\left(\mathrm{L} \cdot \mathrm{min}^{-1}\right)\end{array}$ & $\begin{array}{c}\text { POST- } \\
\dot{V} \mathrm{O}_{2} \text { peak } \\
\left(\mathrm{L} \cdot \mathrm{min}^{-1}\right)\end{array}$ & $\begin{array}{l}\text { PRE-T } \\
\text { (min) }\end{array}$ & $\begin{array}{l}\text { POST- } T_{\text {lim }} \\
\text { (min) }\end{array}$ \\
\hline 1 & 95 & $155^{*}$ & 2.41 & 2.28 & 12.52 & 11.02 \\
\hline 2 & 275 & 275 & 4.07 & 4.20 & 19.01 & 18.76 \\
\hline 3 & 155 & 185 & 3.54 & 3.24 & 15.00 & 15.51 \\
\hline 4 & 185 & $125 * *$ & 3.43 & 2.72 & 14.39 & 13.01 \\
\hline 5 & 185 & 155 & 3.42 & 3.51 & 15.50 & 15.52 \\
\hline 6 & 155 & 125 & 2.47 & 2.29 & 12.49 & 11.52 \\
\hline 7 & 155 & 155 & 2.15 & 2.08 & 11.01 & 11.40 \\
\hline 8 & 125 & 125 & 2.2 & 2.14 & 11.50 & 11.51 \\
\hline 9 & 215 & 245 & 3.61 & 3.72 & 17.51 & 18.40 \\
\hline 10 & 65 & 95 & 2.89 & 3.13 & 14.01 & 15.01 \\
\hline 11 & 65 & 95 & 1.58 & 1.40 & 8.51 & 8.01 \\
\hline 12 & 125 & 155 & 3.01 & 3.27 & 15.00 & 16.50 \\
\hline 13 & 155 & 155 & 2.56 & 2.61 & 14.00 & 13.32 \\
\hline 14 & 155 & 155 & 2.06 & 2.22 & 11.51 & 12.46 \\
\hline 15 & 185 & 185 & 3.31 & 3.46 & 16.00 & 16.51 \\
\hline Mean & $153 \pm$ & $159 \pm$ & $2.85 \pm$ & $2.82 \pm$ & $13.87 \pm$ & $13.90 \pm$ \\
\hline$\pm \mathrm{SD}$ & 54.93 & 49.25 & 0.708 & 0.760 & 2.69 & 3.02 \\
\hline ICC & \multicolumn{2}{|c|}{0.834} & \multicolumn{2}{|c|}{0.944} & \multicolumn{2}{|c|}{0.953} \\
\hline SEM & \multicolumn{2}{|c|}{21} & \multicolumn{2}{|c|}{0.171} & \multicolumn{2}{|c|}{0.61} \\
\hline $\mathrm{MD}$ & \multicolumn{2}{|c|}{58} & \multicolumn{2}{|c|}{0.474} & \multicolumn{2}{|c|}{1.69} \\
\hline
\end{tabular}

*Indicates that the subject had an increase in the PWC $\mathrm{FT}_{\mathrm{FT}}$ that exceeded the minimal difference to be considered a real change (MD). ** Indicates that the subject had a decrease in the $\mathrm{PWC}_{\mathrm{FT}}$ that exceeded the MD. 


\subsection{Mean Group Comparisons}

The one-way ANCOVA for the $\mathrm{PWC}_{\mathrm{FT}}$ values indicated there were no significant differences among groups $(\mathrm{F}=3.141, \mathrm{p}=0.053)$, however pairwise comparisons indicated a significant difference between the CUR and PLA groups $(p=0.016)$, but no differences between FEN and CUR $(p=0.321)$ or FEN and PLA $(p=0.171)$. Therefore, separate one-way ANCOVAs were used to examine the adjusted $\mathrm{PWC}_{\mathrm{FT}}$ means for the PLA vs. CUR $(F=4.906, p=0.035)$ and the PLA vs. FEN $(F=2.969, p=0.097)$. The adjusted $\mathrm{PWC}_{\mathrm{FT}}$ mean $( \pm \mathrm{SD})$ for the CUR $(192.88 \pm 58.41 \mathrm{~W} ; 95 \% \mathrm{CI} 175.98 \mathrm{~W}-$ $209.77 \mathrm{~W})$ was significantly greater $(p=0.035)$ than the PLA $(165.55 \pm 49.25 \mathrm{~W} ; 95 \%$ CI 147.02W-184.07W). The adjusted POST PWCFT means $( \pm S D)$ for the PLA $(166.66 \pm$ 49.25W; 95\% CI 153.14W-180.18W) and FEN (183.22 $31.53 \mathrm{~W}(95 \%$ CI 169.21W197.23W) were not significantly different $(p=0.097)$ (Figure 1 and 2$)$. The one- way ANCOVA for $\dot{V} \mathrm{O}_{2}$ peak $(\mathrm{F}=0.612, \mathrm{p}=0.612)$ and $\mathrm{T}_{\lim }(\mathrm{F}=0.688, \mathrm{p}=0.508)$ indicated there were no significant differences among groups (Figure 3 and 4).

The $3 \times 2$ mixed factorial ANOVAs resulted in no significant group $\mathrm{x}$ time interactions, main effects for group, or main effects for time for total kilocalories, or carbohydrates $(\mathrm{g})$ and protein $(\mathrm{g})$. However, there was a significant main effect for group for fat $(\mathrm{g})(\mathrm{F}=4.327, \mathrm{p}=0.020)$. A follow up one- way ANOVA $(\mathrm{F}=4.327, \mathrm{p}=0.020)$ and between subject t-test indicated that the fat intake (collapsed across time) was greater for FEN compared to PLA $(p=0.015)$ and CUR $(p=0.014)$, but there was no difference between CUR and PLA ( $\mathrm{p}=0.954)$. Supplement compliance was recorded with supplementation logs and demonstrated a mean $( \pm$ SD) compliance rate of $99.5 \% \pm$ $0.51 \%$. 


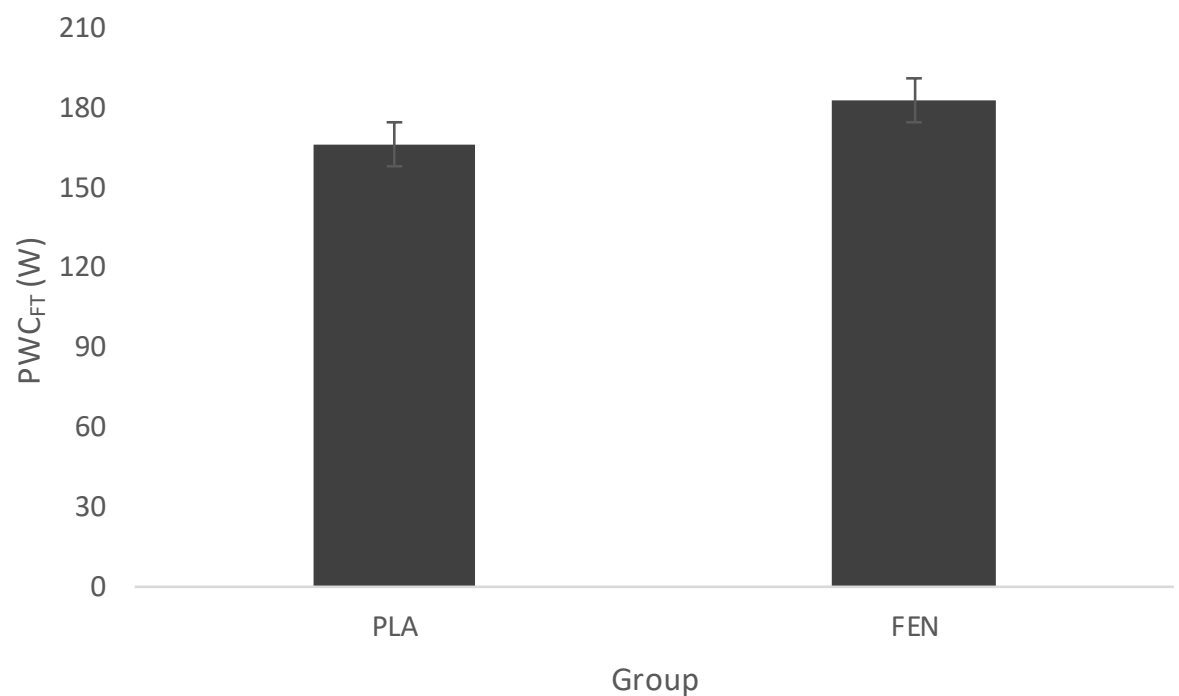

Figure 1. Adjusted POST-test physical working capacity at the fatigue threshold $\left(\mathrm{PWC}_{\mathrm{FT}}\right)$ (mean $\pm \mathrm{SEM}$ ) values (covaried for PRE PWC $\mathrm{FT}$ scores) for the feugreek soluble fiber (FEN) and placebo (PLA) groups.

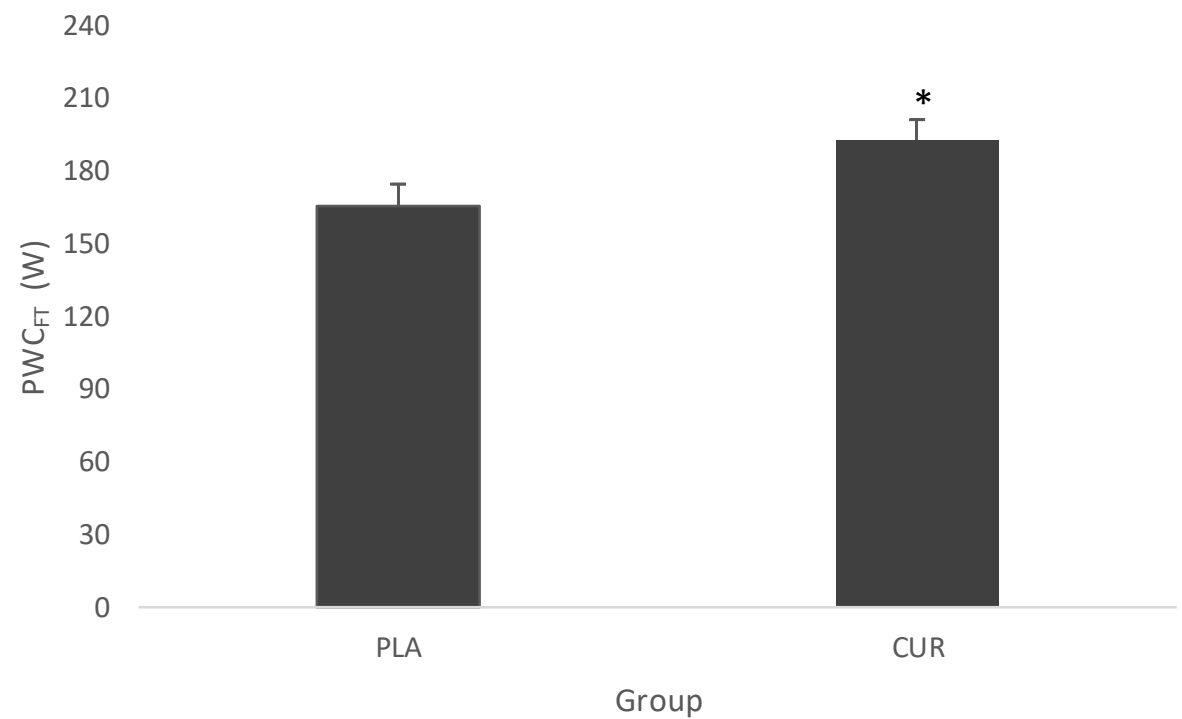

Figure 2. Adjusted POST-test physical working capacity at the fatigue threshold $\left(\mathrm{PWC}_{\mathrm{FT}}\right)$

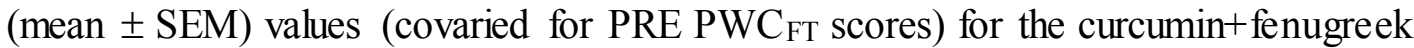
soluble fiber (CUR) and placebo (PLA) groups.

$*$ Significantly $(p<0.05)$ greater than placebo. 


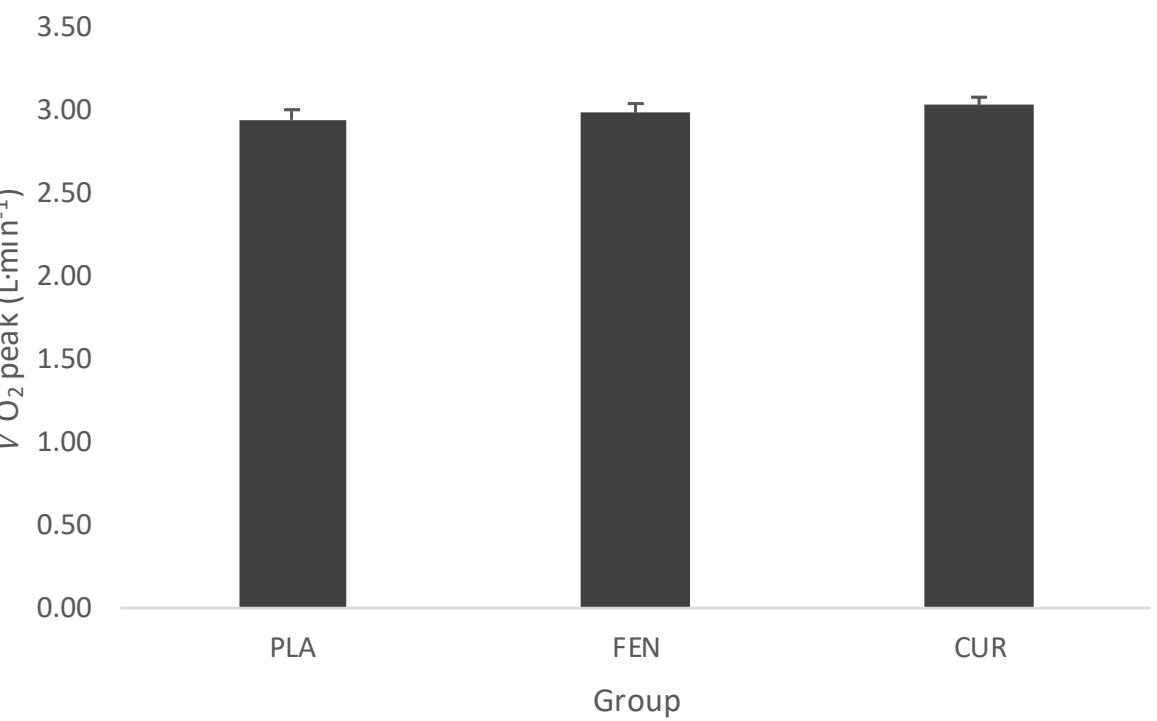

Figure 3. Adjusted POST-test peak oxygen consumption ( $\dot{V}_{2} \mathrm{O}_{2}$ peak) (mean $\pm \mathrm{SEM}$ ) values (covaried for PRE $\dot{V} \mathrm{O}_{2}$ scores) for the curcumin+fenugreek soluble fiber (CUR), fenugreek soluble fiber (FEN), and placebo (PLA) groups.

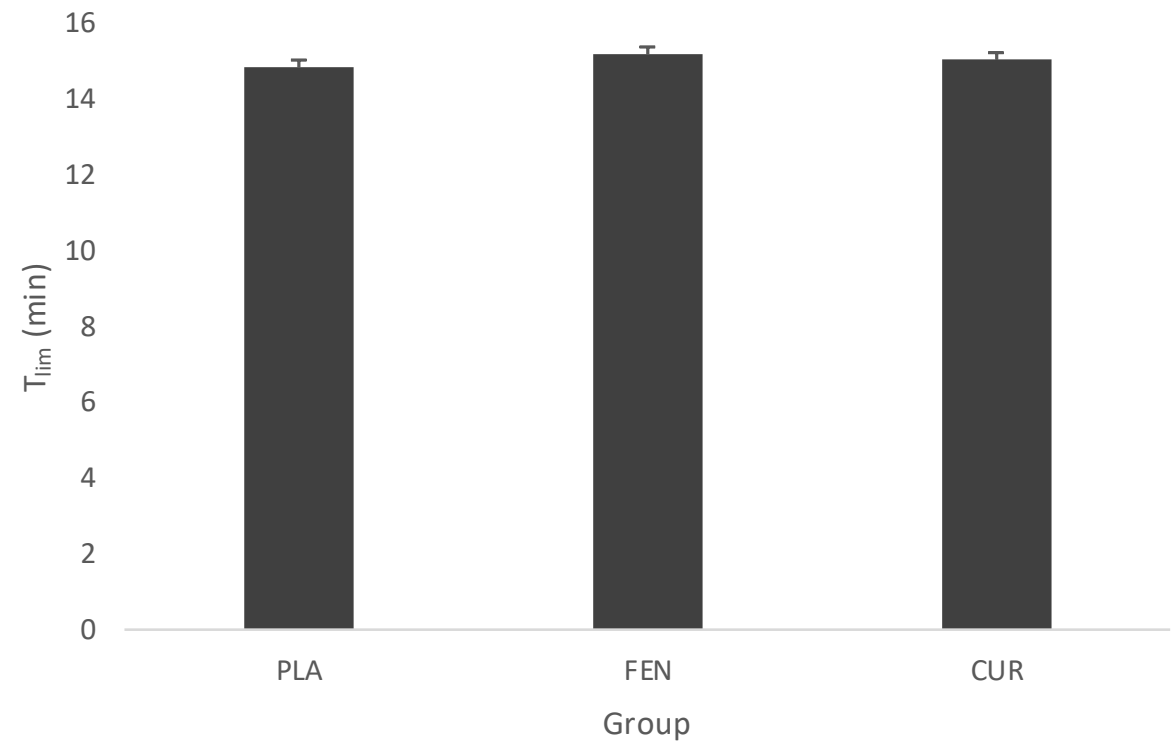

Figure 4. Adjusted POST-test time to exhaustion ( $\left.\mathrm{T}_{\mathrm{lim}}\right)($ mean $\pm \mathrm{SEM}$ ) values (covaried for PRE Tlim scores) for curcumin+fenugreek soluble fiber (CUR), fenugreek soluble fiber (FEN), and placebo (PLA) groups. 


\subsection{Individual Responses}

Four of the 18 subjects in the CUR group (Figure 5), one of the 14 subjects in the FEN group (Figure 6), and one of the 15 subjects in the PLA group (Figure 7) had a value greater than the minimal difference for the PWC $\mathrm{FT}$, but none of the subjects in the PLA, FEN, or CUR groups exceeded the MD for $\dot{V} \mathrm{O}_{2}$ peak or $\mathrm{T}_{\text {lim }}$ (Figures 8-13).

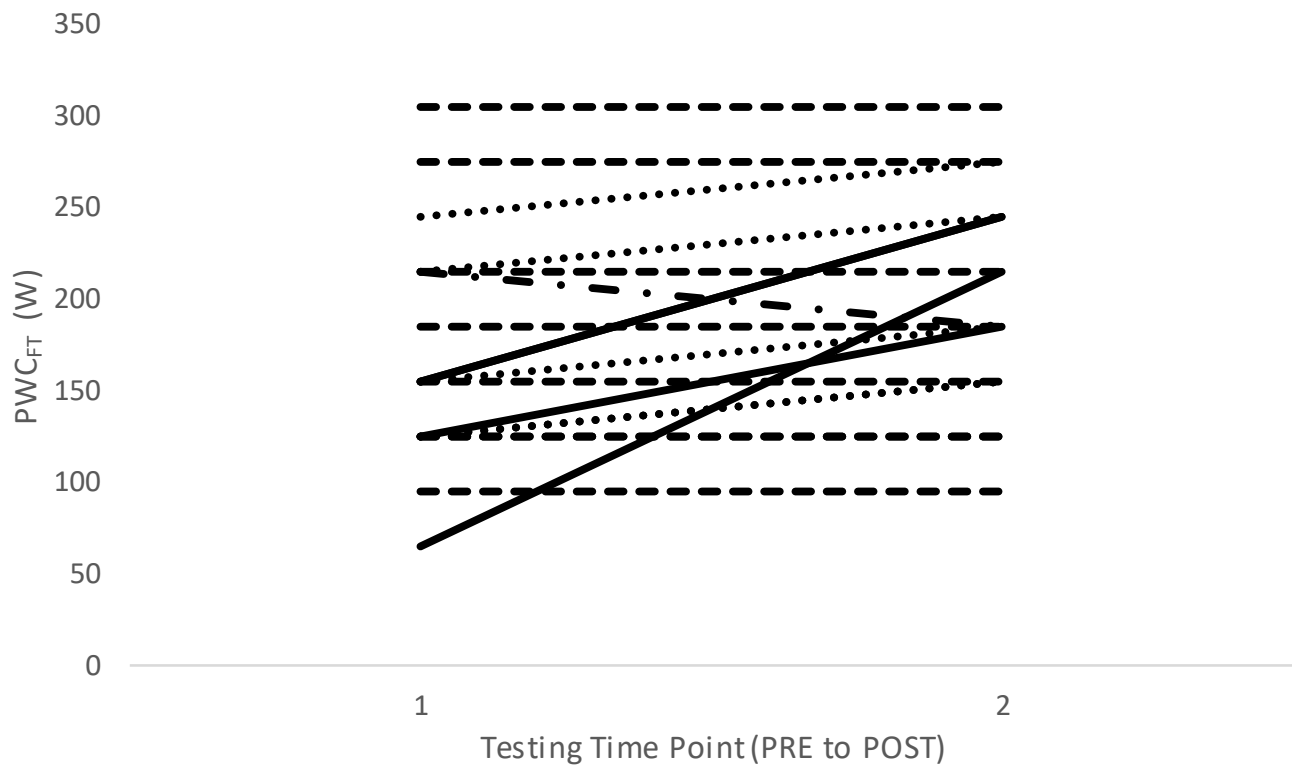

Figure 5. Individual responses for the physical working capacity at the fatigue threshold

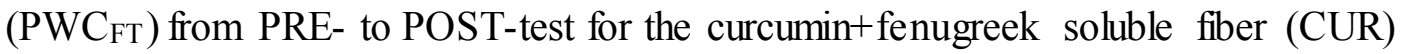
supplement group. Solid line indicates the subject had an increase greater than the minimal difference $(\mathrm{MD}=60 \mathrm{~W})$. Dashed line $(---)=$ no change, dashed and dotted line (- . -) = decrease, and dotted line $(\ldots)=$ increase, not greater than the MD. Note there were 4 subjects who had increases greater than MD, two solid subject lines overlap at 155$245 \mathrm{~W}$. 


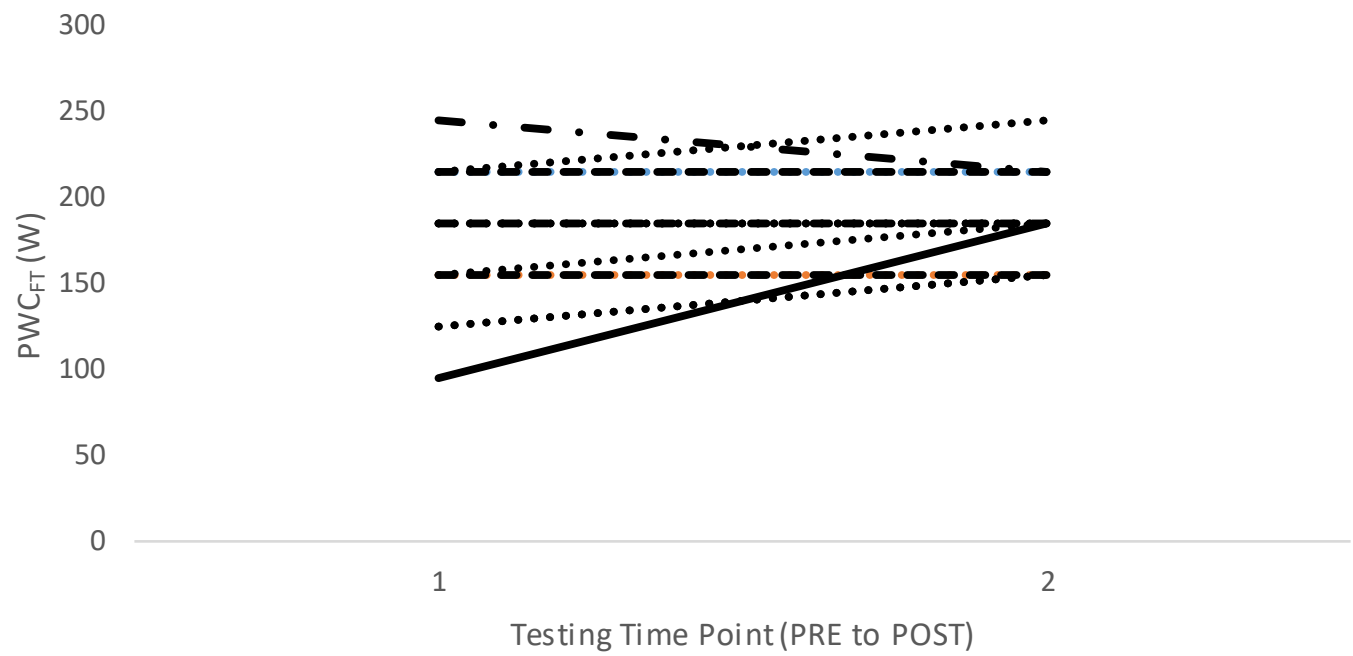

Figure 6. Individual responses for the physical working capacity at the fatigue threshold (PWC group. Solid line indicates the subject had an increase greater than the minimal difference. Dashed line $(---)=$ no change, dashed and dotted line $(-.-)=$ decrease, and dotted line $(\ldots)=$ increase, not greater than the MD.

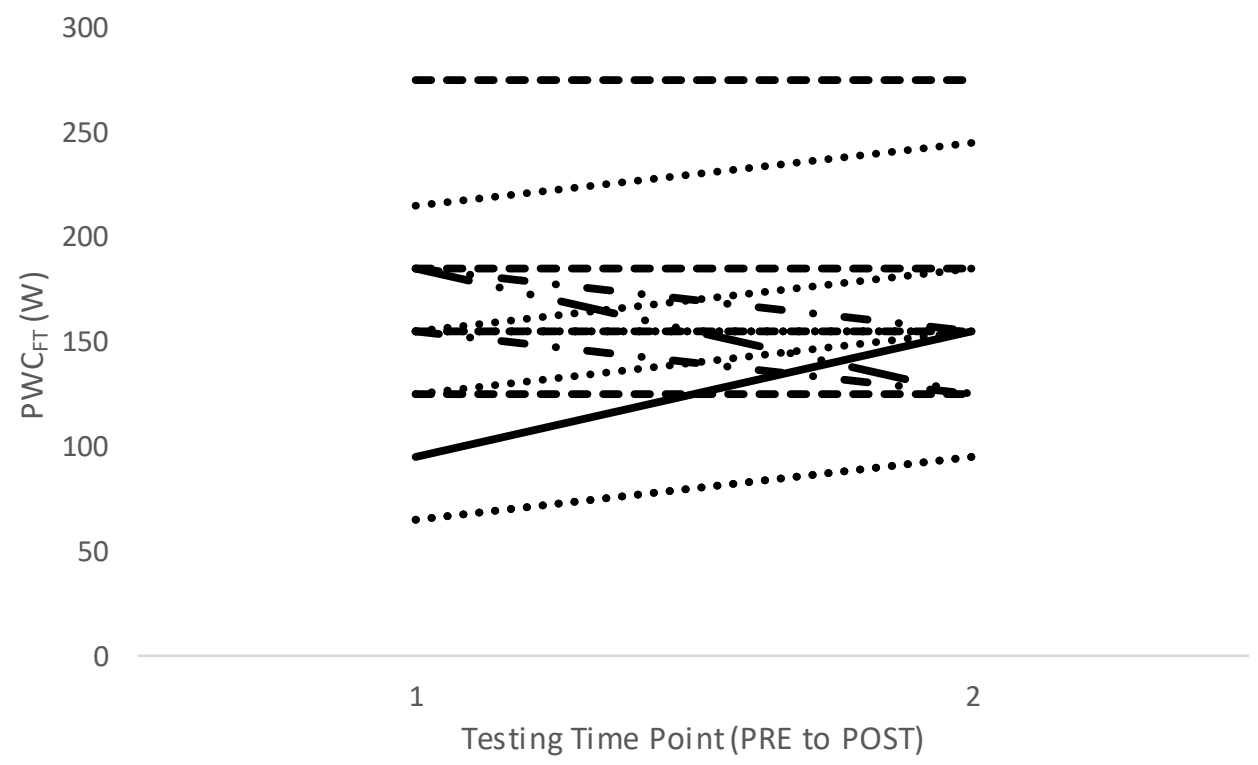

Figure 7. Individual responses for the physical working capacity at the fatigue threshold $\left(\mathrm{PWC}_{\mathrm{FT}}\right.$ ) from PRE- to POST-test for the placebo (PLA) supplement group. Solid line indicates the subject had an increase greater than the minimal difference, dashed, double dot, dashed (-..-) line indicates the subject had a decrease greater than the minimal difference. Dashed line $(---)=$ no change, dashed and dotted line $(-.-)=$ decrease, and dotted line $(\ldots)=$ increase, not greater than the MD. 


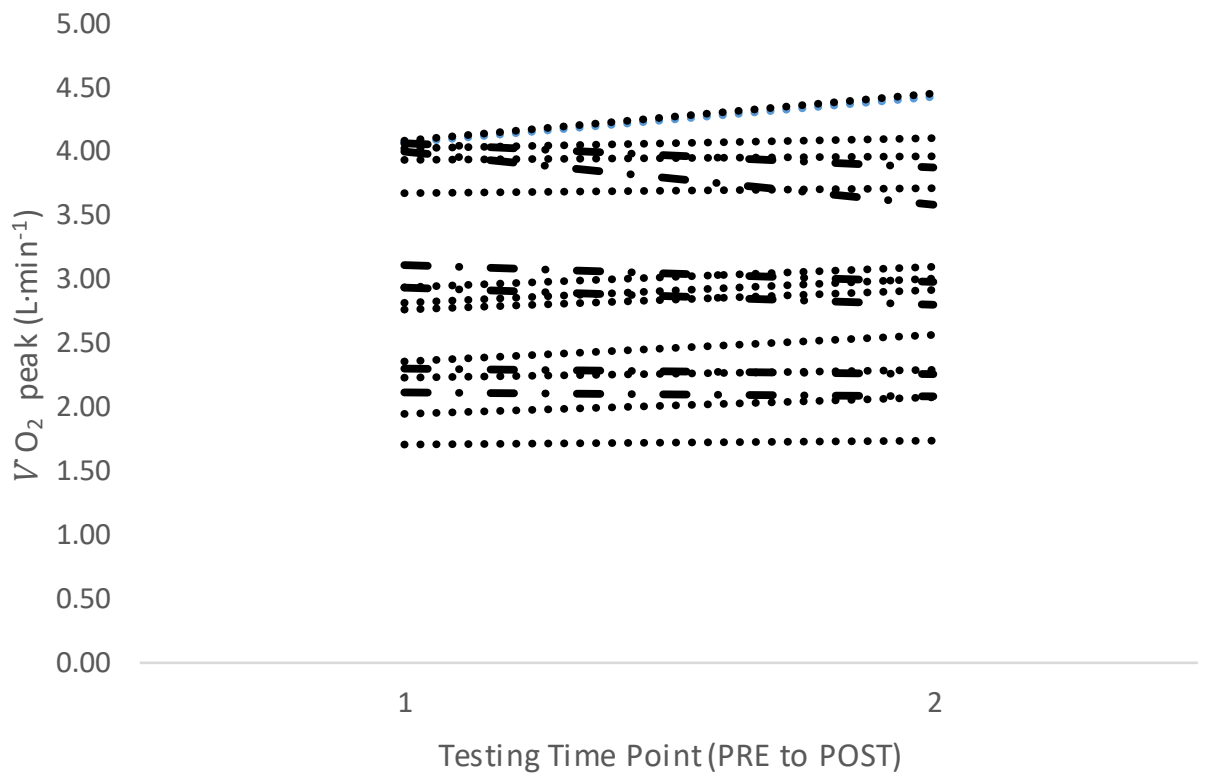

Figure 8. Individual responses for $\dot{V} \mathrm{O}_{2}$ peak from PRE- to POST-test for the curcumin+fenugreek soluble fiber (CUR) supplement group. Solid line indicates the subject had an increase greater than the minimal difference, dashed, double dot, dashed $(-\ldots-)$ line indicates the subject had a decrease greater than the minimal difference. Dashed line $(---)=$ no change, dashed and dotted line $(-.-)=$ decrease, and dotted line $(\ldots)=$ increase.

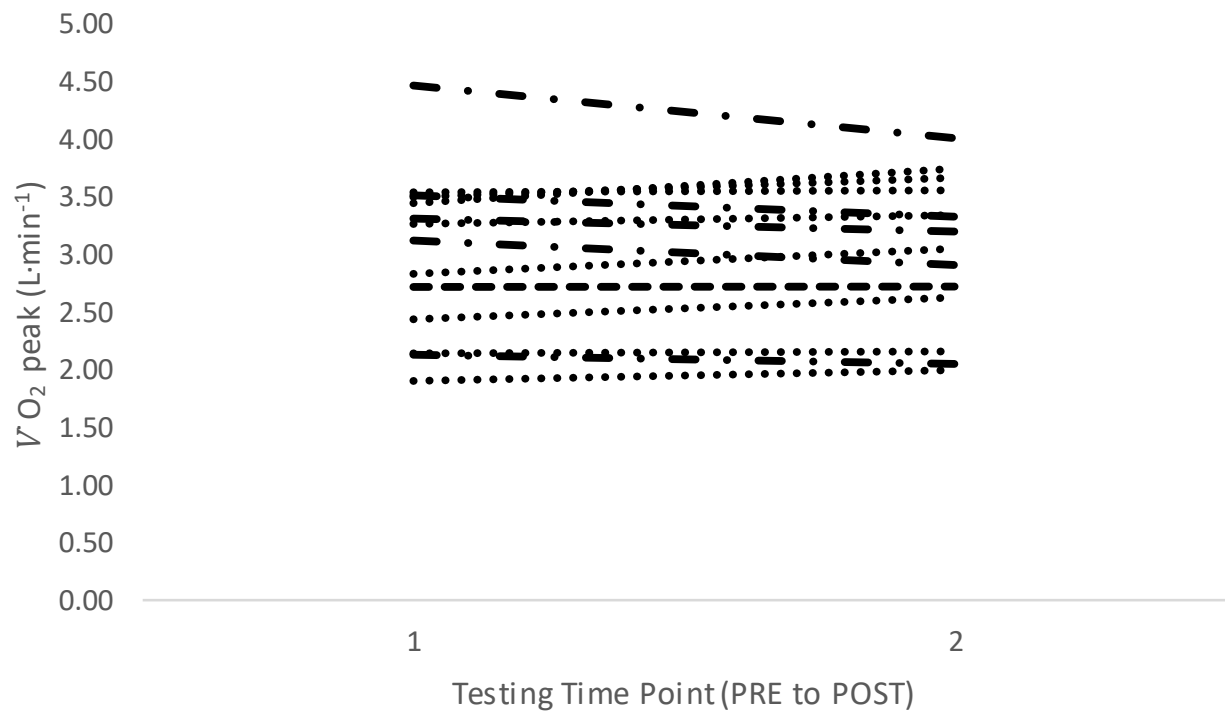

Figure 9. Individual responses for $\dot{V} \mathrm{O}_{2}$ peak from PRE- to POST-test for the fenugreek soluble fiber (FEN) supplement group. Solid line indicates the subject had an increase greater than the minimal difference, dashed, double dot, dashed (- . -) line indicates the subject had a decrease greater than the minimal difference. Dashed line $(---)=$ no change, dashed and dotted line $(-.-)=$ decrease, and dotted line $(\ldots)=$ increase. 


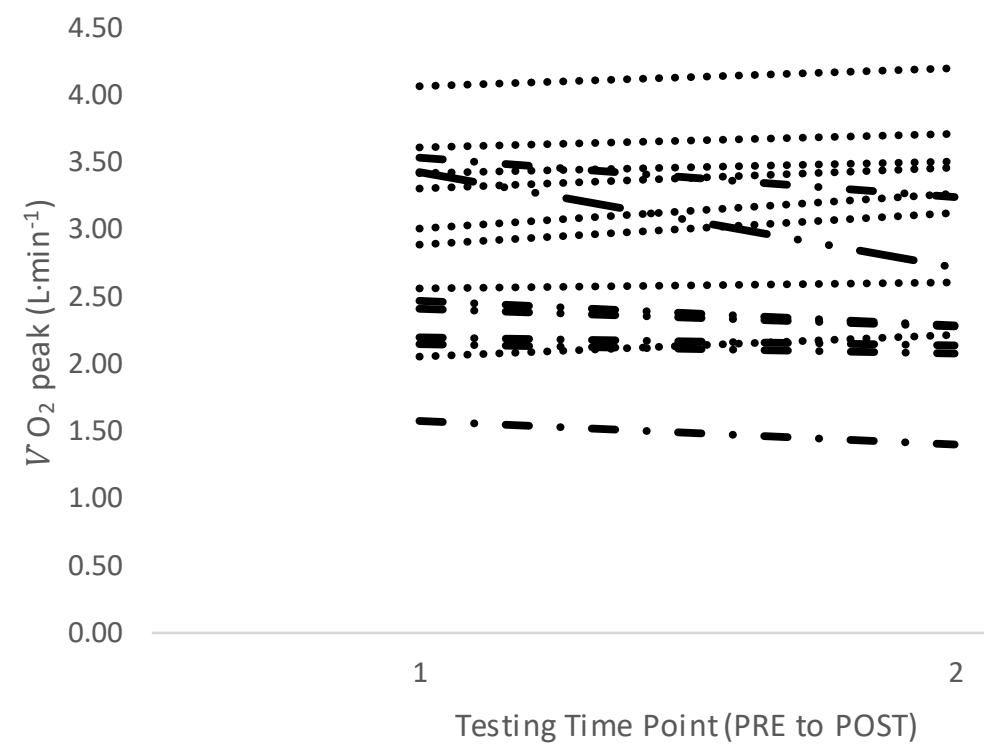

Figure 10. Individual responses for $\dot{V} \mathrm{O}_{2}$ peak from PRE- to POST-test for the placebo (PLA) supplement group. Solid line indicates the subject had an increase greater than the minimal difference, dashed, double dot, dashed (- . .) line indicates the subject had a decrease greater than the minimal difference. Dashed line $(---)=$ no change, dashed and dotted line $(-.-)=$ decrease, and dotted line $(\ldots)=$ increase.

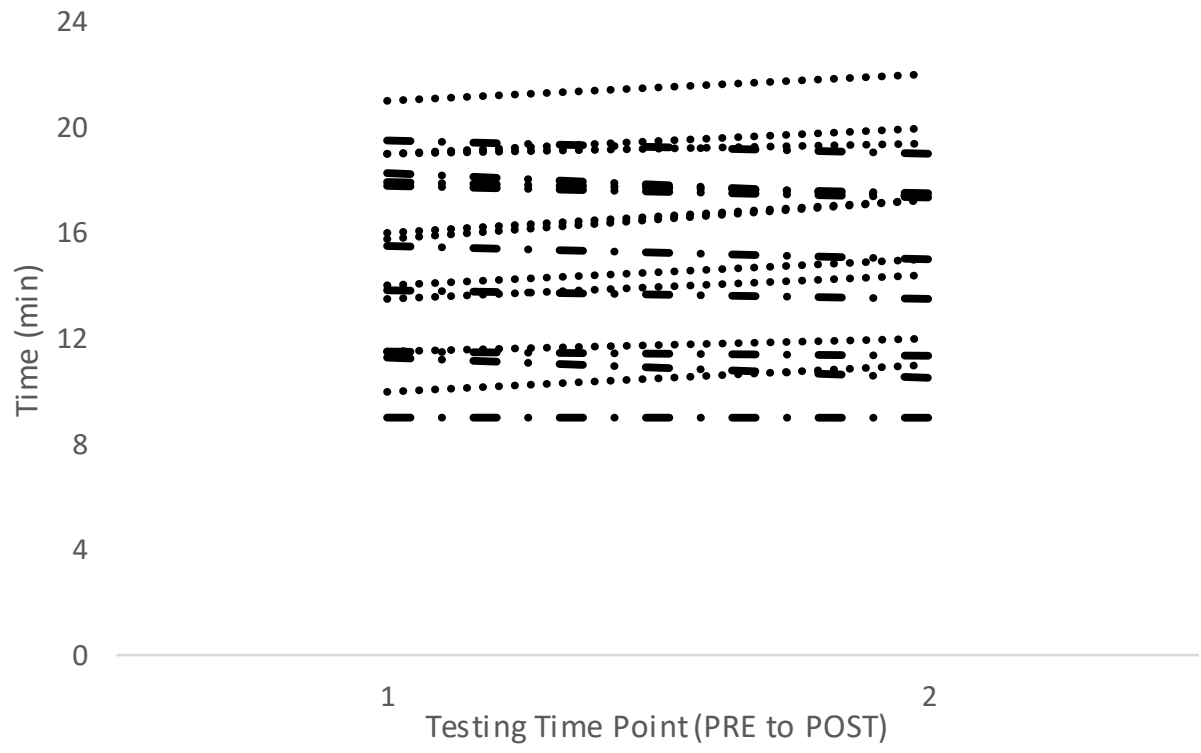

Figure 11. Individual responses for time to exhaustion from PRE- to POST-test for the curcumin+fenugreek soluble fiber (CUR) supplement group. Solid line indicates the subject had an increase greater than the minimal difference, dashed, double dot, dashed (...-) line indicates the subject had a decrease greater than the minimal difference. Dashed line $(---)=$ no change, dashed and dotted line $(-.-)=$ decrease, and dotted line $(\ldots)=$ increase. 


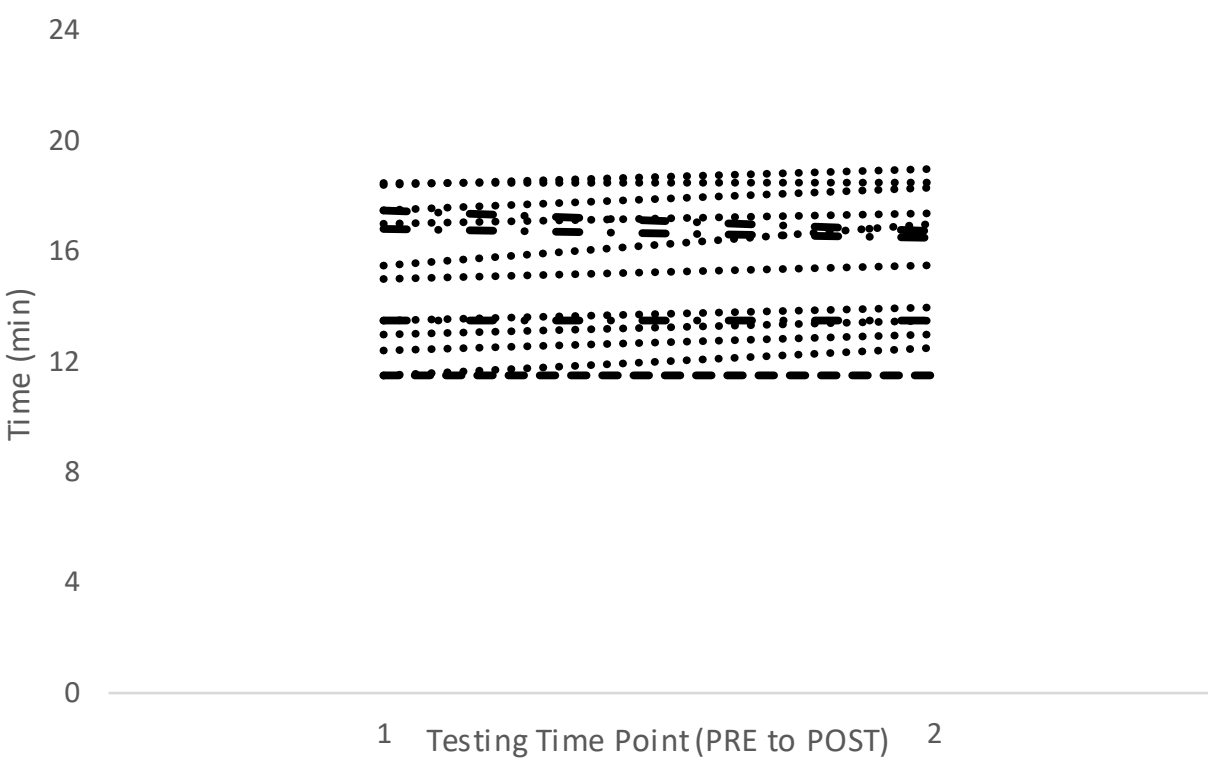

Figure 12. Individual responses for time to exhaustion from PRE- to POST-test for the fenugreek soluble fiber (FEN) supplement group. Solid line indicates the subject had an increase greater than the minimal difference, dashed, double dot, dashed (-..-) line indicates the subject had a decrease greater than the minimal difference. Dashed line (---) $=$ no change, dashed and dotted line $(-.-)=$ decrease, and dotted line $(\ldots)=$ increase.

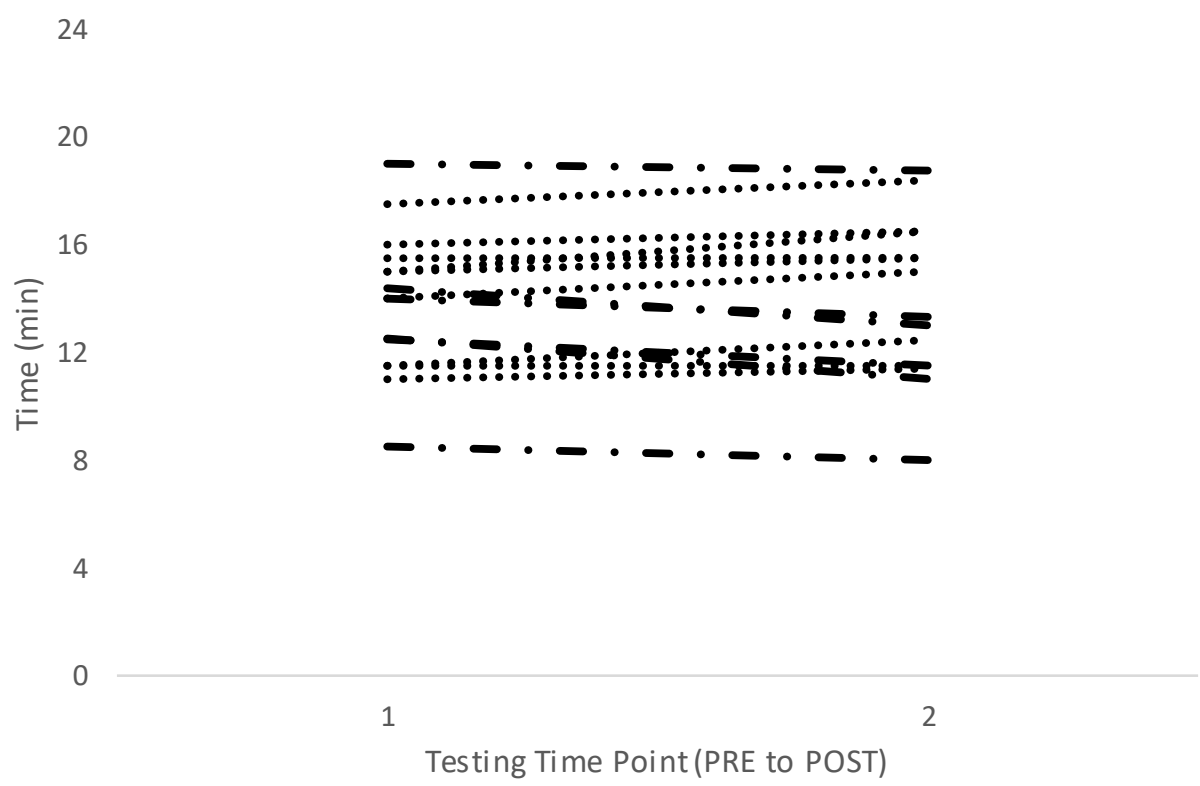

Figure 13. Individual responses for time to exhaustion from PRE- to POST-test for the placebo (PLA) supplement group. Solid line indicates the subject had an increase greater than the minimal difference, dashed, double dot, dashed (- . - ) line indicates the subject had a decrease greater than the minimal difference. Dashed line $(---)=$ no change, dashed and dotted line $(-.-)=$ decrease, and dotted line $(\ldots)=$ increase. 


\section{CHAPTER 5. DISCUSSION}

The primary findings of the current study were that 28 days of curcumin+fenugreek soluble fiber (CUR) and fenugreek soluble fiber (FEN) supplementation did not affect $\dot{\mathrm{VO}}_{2}$ peak or $\mathrm{T}_{\text {lim, }}$, but the PWC $\mathrm{PT}_{\mathrm{F}}$ was greater at POST-test for the CUR, compared to the PLA group. In addition, there was no difference in the $\mathrm{PWC}_{\mathrm{FT}}$ at POST-test between

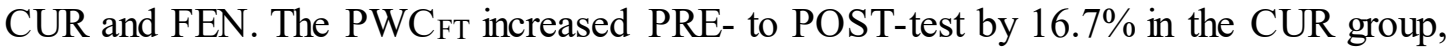
$8.5 \%$ in the FEN group, and only by $3.9 \%$ in the PLA group. The increase in the PWC $\mathrm{FT}$ for the CUR group in this study were consistent with the $22.4 \%$ and $18.8 \%$ increases in the PWC $\mathrm{FT}_{\mathrm{FT}}$ from PRE- to POST-test previously reported (Camic et al. 2010) after 4 weeks of daily oral administration of arginine-based supplements (Camic et al. 2010). Like arginine, one of the potential physiological actions of curcumin is its role as a precursor to nitric oxide (NO) production, which plays an important role in endotheliumdependent vasodilation (Boonla et al. 2014). Thus, the changes in the PWCFT in the CUR supplementation group in the present study were consistent with the changes previously reported following 28 days of a nutritional supplement intervention without exercise training, and may be related to increased blood flow and metabolic byproduct clearance, triggered by increased NO bioavailability, a potent vasodilator (Camic et al 2010).

The changes observed for the PWC $\mathrm{FT}_{\mathrm{F}}$ for the CUR group in this study were likely related to the factors that underlie neuromuscular fatigue and the detection of the threshold. The $\mathrm{PWC}_{\mathrm{FT}}$ is an electromyographic (EMG) technique that theoretically estimates the highest power output that can be maintained for an extended period of time without evidence of neuromuscular fatigue. Previous studies have examined the potential 
mechanisms underlying neuromuscular fatigue, which are reflected by increases in the EMG signal and include additional motor unit recruitment and/or increases in firing rate of the initially recruited motor units to maintain exercise at a constant power output (Camic et al 2010, Zuniga et al). For example, it has been suggested (Camic et al 2010, Zuniga et al 2010, Bergstrom et al 2013) that the fatigue-induced changes in EMG amplitude may be related to the accumulation of various metabolic byproducts and/or ions (i.e., inorganic phosphate, hydrogen ions, ammonia and potassium). Further studies have shown that the accumulation of these metabolites and ions result in fatigue-induced decreases in membrane excitability (Clausen 2013, Enoka and Stuart 1992), excitationcontraction coupling involving $\mathrm{Ca}++$ release and uptake from the sarcoplasmic reticulum, myofibrillar $\mathrm{Ca}++$ sensitivity for binding with troponin, actin and myosin binding, and ATP production and breakdown (Gladden 2004, MacLaren et al 1989, Robergs et al 2004, Westerblad et al 2002). Other studies attribute the recruitment of additional motor units to maintain force or power output during a fatiguing task to the decrease in intracellular $\mathrm{pH}$ as a result of non-mitochondrial ATP hydrolysis, which contributes to the decline in muscle fiber conduction velocity (Lindstrom et al 1970, Moritani et al 1982). It has been hypothesized (Camic et al 2010) that, during incremental cycle ergometry, intracellular $\left[\mathrm{H}^{+}\right]$may rise to a critical level, decreasing intracellular $\mathrm{pH}$, and interfering with excitation-contraction coupling. These effects may result in fatigue changes in induced motor unit activation and an increase in EMG amplitude observed at the PWCFT.

The physiological mechanisms responsible for the ergogenic effects of curcumin have not been fully identified, but some reported effects include increased metabolic 
byproduct clearance (Sahin et al 2016, Huang et al 2015), potentially linked to increased NO bioavailability and the subsequent vasodilatory response (Boonla et al 2014), as well as increased blood antioxidant capacity, and reduced oxidative stress during exercise from decreased levels of reactive oxygen species (ROS) (Takahashi et al 2013). Curcumin is an active ingredient of polyphenolic curcuminoids, which can be found in the spice turmeric and has been well studied in clinical investigations over the last few decades (Boonla et al 2014). It is possible the purported (Boonla et al 2014, Sahin et al 2016, Takahashi et al 2013) effects of curcumin supplementation to increase clearance of metabolic byproducts through improved endothelial function, increased neuromuscular time to fatigue and the $\mathrm{PWC}_{\mathrm{FT}}$ in this study. For example, curcumin has been shown to decease the accumulation of metabolic byproducts such as lactate and ammonia (Sahin et al 2016), metabolites that have previously been linked to neuromuscular fatigue as they accumulate during repeated muscle contractions (Camic et al 2010, Zuniga et al 2010, Bergstrom et al 2013). Thus, it is possible curcumin improves clearance of metabolic byproducts in the muscle cells through NO mediated vasodilation may increase the time to neuromuscular fatigue, and allow the working muscle to perform at a higher power output without the changes in muscle activation, thus delaying the PWC

In the present study, fenugreek soluble fiber was added to increase the bioavailability of curcumin (Krishnakumar et al 2012), but it may also have physiological effects. Fenugreek is a naturally growing plant, originating in India and Northern Africa (Srichamroen et al 2008). The seeds and leaves have been used for hundreds of years in Ayurvedic and Chinese medicine, however recently, preliminary animal and human trials suggest that oral fenugreek seed powder may have possible hypoglycemic and 
antihyperlipidemic properties (Basch et al 2003). In the present study, the galactomannan component of fenugreek was used in the CUR supplement to increase absorption from the small intestine (Krishnakumar et al 2012). Galactomannan is a soluble fiber guar gum, and a potential active component of fenugreek seeds (Srichamroen et al 2008). Specifically, galactomannans from fenugreek have been shown to have effects on glycemic and lipidemic status in rats (Srichamroen et al 2008). Specifically, galactomannan- fed rats demonstrated significantly less adipose tissue compared to the control, suggestive of a high metabolic rate in the tissue that were also associated with increased release of FFA into the circulation and decreased plasma insulin levels (Srichamroen et al 2008). The authors suggested that the reduction of plasma insulin level in galactomannan-fed rats may increase lipolysis in adipose tissue, and consequently increase plasma FFA levels and reduce adipocyte size (Srichamroen et al 2008). Thus, it is possible the fenugreek component in the CUR and FEN groups increased FFA release and decreased insulin levels. These factors may have contributed to the increased neuromuscular time to fatigue for the CUR group by delaying the reliance on anaerobic energy production and accumulation of metabolic byproducts from glucose metabolism. This may also explain, in part, the non-significant difference between the CUR and FEN group at POST-test.

In this study, it appears the purported effects of curcumin to increase NO production and aid in metabolic byproduct clearance were effective to increase the submaximal neuromuscular fatigue threshold $\left(\mathrm{PWC}_{\mathrm{FT}}\right)$, but did not affect indices of maximal performance such as $\mathrm{T}_{\lim }$ and $\mathrm{VO}_{2}$ peak. It is possible the intensity specific effects were related to the factors affecting $\dot{\mathrm{VO}}_{2}$ peak and the mode of exercise to 
determine $T_{\text {lim. }}$ Previous investigators (Sahin et al. 2016) have reported decreased levels of lactate, ammonia, and muscle malondialdehyde in conjunction with increased run times to exhaustion in rodents supplemented with curcumin compared to a placebo. Consistent with the findings by Sahin et al. (2016), Huang et al. (2015) reported that curcumin supplementation dose-dependently increased grip strength and endurance performance and increased muscular glycogen content and reduced levels of physical fatigue-associated biomarkers serum lactate, ammonia, and blood urea nitrogen compared to the vehicle treatment (Huang et al 2015). Each of these previous endurance tests in animal models used submaximal protocols to examine changes in $\mathrm{T}_{\lim }$ (Sahin et al 2016, Huang et al 2015). In the present study, however, a maximal GXT on a bicycle ergometer was utilized, which may not have been sensitive to the physiological changes induced by curcumin, due to the observed non-significant increases in time PRE to POST. Specifically, atthough improved metabolic byproduct clearance may have delayed neuromuscular fatigue and the $\mathrm{PWC}_{\mathrm{FT}}$, an increase in Tlim for the GXT would likely be dependent factors that increased the cellular and blood buffering capacities as well as improved cellular respiration at maximal intensities. Thus, the results of the present study indicated curcumin and fenugreek soluble fiber were effective to delay neuromuscular

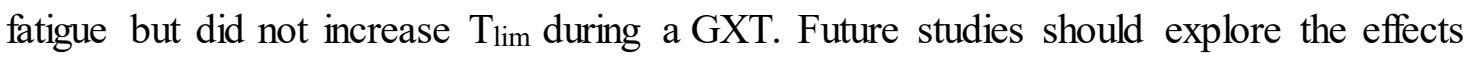
of curcumin on the sustainability of submaximal exercise performed at or below the $\mathrm{PWC}_{\mathrm{FT}}$ to determine if the factors that improved the threshold might also increase $\mathrm{T}_{\text {lim. }}$

Similar to $\mathrm{T}_{\text {lim, }}$, there were no effects of supplementation on $\mathrm{V}_{2}$ peak in the present study. Maximal oxygen uptake ( $\mathrm{VO}_{2}$ peak) is primarily limited by the ability of the cardiorespiratory system to deliver oxygen to the exercising muscles (Basset et al 
2000), but is also dependent on peripheral factors related to skeletal muscle $\mathrm{O}_{2}$ extraction such as capillary and mitochondrial density (Basset et al 2000), which play a critical role in improving submaximal endurance performance. Of the many factors which may increase michondrial biogenesis, effects of endurance training have been well studied and shown to cause an increase in mitochondrial enzyme activities, which improves performance by enhancing fat oxidation and decreasing lactic acid accumulation at a given $\dot{\mathrm{VO}}_{2}$ (Zamora et al 1995). A study by Zamora et al (1995) found that following 10 weeks of endurance training in men there was a significant increase in the number of mitochondria per area and in the relative surface occupied by the total mitochondria profile area. In the present study, it appears 28 days of curcumin supplementation was not effective to alter physiological mechanisms which would lead to increases in $\mathrm{V}_{2}$ peak, however previous research has demonstrated cellular effects of curcumin supplementation in mice that were amplified with exercise (Hamidie et al 2015). Specifically, the combination of curcumin supplementation and endurance training has the potential to accelerate mitochondrial biogenesis in skeletal muscle by increasing cAMP levels (Hamidie et al 2015). In the present study, subjects were only supplemented with CUR for 28 days, during which time they were instructed not to change dietary or exercise habits. Without an increase in endurance training during supplementation time, it is possible that CUR supplementation alone did not have potent enough effects to increase cardiac output, which, theoretically, would result in an increase of $\mathrm{VO}_{2}$ peak. Additionally, according to Basset et al (2000), peripheral cellular changes have been shown to improve submaximal performance. Therefore, any potential increases in capillary and mitochondrial density as a result from curcumin supplementation may have 
had an effect on the $\mathrm{PWC}_{\mathrm{FT}}$, a submaximal work rate, but not $\mathrm{VO}_{2}$ peak, which represents maximal exercise. Future studies should aim to investigate whether a longer supplementation period, or higher dosages of curcumin may lead to increases in $\mathrm{VO}_{2}$ peak. Furthermore, curcumin supplementation with endurance training should be investigated, to determine if the combination of the two interventions may lead to an increase in $\dot{\mathrm{VO}}_{2}$ peak.

In the present study, a reliability analysis was used observe individual responses and establish the MD, to determine the magnitude of change in PRE- to POST-test values necessary to be considered real (Weir 2005). The MD speaks to the sensitivity of the test in distinguishing a "real" change from variation or error in measurement (Weir 2005). The MD for the change to be real for the PWC $\mathrm{FT}$ was $60 \mathrm{~W}$ in this study, which reflected a delay in neuromuscular fatigue by two stages from PRE to POST-test. In the PLA group, one of the 15 subjects had a change greater than the MD, and one subject had a change that was less than the MD ( $\sim 7 \%$ of the subjects). In the FEN group, one of the 14 subjects exceeded the MD (7\% of the subjects), and four out of the 18 exceeded the MD ( $22 \%$ of the subjects) in the CUR group. Thus, in general the individual subject responses in the CUR group were not necessarily reflective of the mean response for the CUR group. Therefore, the mean difference at POST-test for the CUR was likely driven by a few high responders (exceeded the MD) and more moderate changes for the remaining subjects (Figures 5-13). Extended to a larger population, 4-weeks of supplementation with curcumin may only be effective for $\sim 20 \%$ of individuals. It is possible that the subjects who showed improvement in this study had more potential for change, which predisposed them to be more sensitive to actions of curcumin supplementation. Factors 
that may impact an individual's ability to respond to supplementation include prior training status, health history, and potential genetic factors (Roberts et al 2018). Of these genetic factors, Bamman et al. (2007) reported that different skeletal muscle biomarkers exist between high responders, who showed a marked increase in muscle fiber crosssectional area (fCSA), relative to low responders following 16 weeks of resistance training. A proposed mechanism for this difference in high versus low responders is the difference in levels of skeletal muscle insulin-like growth factor-1 (IGF-1) mRNA variants as well as an mRNA indicative of satellite cell differentiation (myogenin). These markers were expressed higher in the high responders relative to low responders following training (Bamman et al 2007). To date, there is limited evidence whether there are high responders versus low responders to nutritional supplements, and the underlying mechanisms. Future studies should aim to investigate factors affecting a subject's response to nutritional supplementation. Although the subjects in this study reported training frequencies and volume consistent with moderately trained, college aged individuals, there was a wide range of cardiorespiratory fitness levels (very poor to superior) based on $\dot{\mathrm{V}}{ }_{2}$ peak values measured from the GXT (ACSM's Guidelines for Exercise Testing and Prescription). In addition, subjects were eliminated from participation if they had a history of cardiovascular or respiratory illnesses, however, no additional measures of health status were taken beyond resting heart rate and blood pressure. Previous investigators have observed the effects of curcumin on arterial stiffness in mice due to aging and concluded that curcumin supplementation improves large elastic arterial stiffening as a result of aging, NO-mediated vascular endothelial dysfunction, decreases oxidative stress and increases collagen and advanced glycation 
end products (AGEs) in mice (Fleenor et al 2013). It is possible that the subjects who demonstrated significant improvements after supplementation were more sensitive to change due to factors like arterial stiffness. Future studies should examine cardiovascular health measures and prior training status to determine if these factors are related to the potential for an individual to respond to curcumin supplementation.

Potential limitations of this study may be the result of the inability of to consistently monitor subject's actives and habits over the duration of the study. To ensure compliance, subjects were given only 7 days of the supplement at a time, along with a dosing log and were required to check in with investigators weekly to report if they had missed any doses before receiving their next supply. Subjects were only included in the study if they were $80 \%$ compliant with their supplementation (compliancy $=(\#$ of doses consumed / total \# of doses provided) x 100), however, there was no measure included to determine levels of curcumin in the circulation. Furthermore, to rule out the potential effects of exercise or nutrition on the results, the subjects kept an exercise log over the 28 days as well as a food log for the three days leading up to the PRE-and POST tests and were instructed not to change dietary or exercise habits. Lastly, other factors that may affect performance, such as sleep, and study habits were not measured and quantified, which could have an effect if changed or disrupted over the course of the study.

In conclusion, $\mathrm{CurQ}$ fen ${ }^{\circledR}$ supplementation increased the $\mathrm{PWC}_{\mathrm{FT}}$ compared to a placebo, but not compared to fenugreek alone. However, there were no effects of CurQfen巴 on $\dot{V}_{2}$ peak or $T_{\text {lim. }}$. The mechanisms responsible for delaying time to neuromuscular fatigue may include increased NO production, and increased blood flow to remove metabolic byproducts. Considering individual responses, CurQfen® 
supplementation resulted in a real change in the $\mathrm{PWC}_{\mathrm{FT}}$ for a small portion of the subjects $(\sim 20 \%)$. These findings suggested that CurQfen ${ }^{\circledR}$ supplementation without exercise training may help to improve time to neuromuscular fatigue in certain individuals, but the group mean analyses were not necessarily reflective of the responses for a majority of the subjects. 


\section{REFERENCES}

Abe T, Kumagai K, Brechue WF (2000) Fascicle length of leg muscle is greater in sprinters than distance runners. Med Sci Sport Exerc 32:1125-1129

Bamman, M M., Petrella, J K., Kim, J S., Mayhew, D L., and Cross, J M. (2007). Cluster analysis tests the importance of myogenic gene expression during myofiber hypertrophy in humans. J. Appl. Physiol. 102, 2232-2239

Basch E, Ulbricht C, Kuo G, Szapary P, Smith M (2003). Therapeutic Applications of Fenugreek. Alternative Medicine Review. Volume 8, Number 1:20-27

Basset D R Jr., Howley E T (2000). Limiting factors for maximum oxygen uptake and determinants of endurance performance. Med Sci Sports Exerc. 32(1):70-84

Bergstrom H C, Housh T J, Cochrane K C, Jenkins N D M1, Lewis R W Jr, Traylor D A, Zuniga J M 2, Schmidt J R, Johnson G O, Cramer J T (2013) An examination of neuromuscular and metabolic fatigue thresholds. Physiol. Meas. 34 (2013) 1253-1267 Boonla O, Kukongviriyapan U, Pakdeechote P, Kukongviriyapan V, Pannangpetch P, Prachaney P, Greenwald E S (2014). Curcumin improves endothelial dysfunction and vascular remodeling in $2 \mathrm{~K}-1 \mathrm{C}$ hypertensive rats by raising nitric oxide availability and reducing oxidative stress. 
Camic L C, Housh T J, Johnson G O, Hendrix C R, Zuniga J M, Mielke M and Schmidt R J (2010a). An EMG frequency-based test for estimating the neuromuscular fatigue threshold during cycle ergometry Eur. J. Appl. Physiol. 108 337-45

Camic L C, Housh T J, Zuniga J M, Hendrix C R, Mielke M, Johnson G O and Schmidt R J (2010b) Effects of arginine based supplements on the physical working capacity at the fatigue threshold J. Strength Cond. Res. 24 1306-12

Clausen T 2013 Excitation-induced exchange of $\mathrm{Na}+, \mathrm{K}+$, and $\mathrm{Cl}-$ in rat EDL muscle in vitro and in vivo: physiology and pathophysiology J. Gen. Physiol. 141 179-92

Davis J M, Murphy E A, Carmichael M D, Zielinski M R, Groschwitz C M, Brown A S, Gangemi J D, Ghaffar A, Mayer E P (2007). Curcumin effects on inflammation and performance recovery following eccentric exercise-induced muscle damage. Am J Physiol Regul Integr Comp Physiol. 2007 Jun; 292(6): R2168-73

deVries H A, Moritani T, Nagata A, Magnussen K (1982). The relation between critical power and neuromuscular fatigue as estimated from electromyographic data. The Journal of Ergonomics.

deVries H A, Tichy W M, Housh J T, Smyth D K, Tichy M A, Housh J D (1987). A method for estimating physical working capacity at the fatigue threshold $\left(\mathrm{PWC}_{\mathrm{FT}}\right)$. The Journal of Ergonomics. 
deVries H A, Housh J T, Johnson O G, Evans A S, Tharp D G, Housh J D, Hughes A R (1990). Factors affecting the estimation of physical working capacity at the fatigue threshold. The Journal of Ergonomics.

Enoka R M and Stuart D G (1992). Neurobiology of muscle fatigue J. Appl. Physiol. 72 $1631-48$

Fleenor B S, Sindler A L, Marvi N K, Howell K L, Zigler M L, Yoshizawa M, Seals D R (2013). Curcumin Ameliorates Arterial Dysfunction and Oxidative Stress with Aging. Exp Gerontol. 48(2):269-76.

Gladden L B (2004). Lactate metabolism: a new paradigm for the third millennium J. Physiol. 558 5-30

Hamidie R D R, Yamada T, Ishizawa R, Saito Y, Masuda K (2015). Curcumin treatment enhances the effect of exercise on mitochondrial biogenesis in skeletal muscle by increasing cAMP levels.

Hermens H J, Freriks B, Merletti R, Stegeman D, Blok J, Rau G, Disselhorst-Klug C, Hägg G (1999) Seniam 8: European recommendations for surface electromyography: results of the seniam project. Roessingh Research and Development

Huang W C, Chiu W C, Chuang H L, Tang D W, Lee Z M, Wei L, Chen F A, Huang C C (2015). Effect of Curcumin Supplementation on Physiological Fatigue and Physical Performance in Mice. Nutrients 2015, 7, 905-921 
Krishnakumar I M, Ravi A, Kumar D, Kuttan R, Maliakel B (2012). An enhanced bioavailable formulation of curcumin using fenugreek-derived soluble dietary fibre. JOURNAL OF FUNCTIONAL FOODS 4 (2012) 348-357

Kwatny E D, Thomas D H, Kwanty H G (1970) An application of signal processing techniques to the study of myoelectric signals. IEEE Trans Biomed Eng 17:303-313 Lindstrom L, Magnusson R, Petersen I (1970). Muscular fatigue and action potential conduction velocity changes studied with frequency analysis of EMG signals. Electromyography $10: 341-356$

MacLaren DP, Gibson H, Parry-Billings M, Edwards RH (1989). A review of metabolic and physiological factors in fatigue. Exerc Sport Sci Rev 17:29-66

Moritani T, Nagata A, Muro M (1982) Electromyographic manifes- tations of muscular fatigue. Med Sci Sport Exerc 14:198-202

Malek M H, Housh T J, Coburn J W, Weir J P, Schmidt R J, Beck T W (2006) The effects of interelectrode distance on electromyographic amplitude and mean power frequency during incremental cycle ergometry.. J Neurosci Methods 151:139-47

Robergs R A, Ghiasvand F and Parker D (2004). Biochemistry of exercise-induced metabolic acidosis Am. J. Physiol. Regul. Integr. Comp. Physiol. 287 R502-16

Roberts M D, Haun C T, Mobley C B, Mumford P W, Romero M A, Roberson P A, Vann C G, McCarthy J J (2018). Physiological Differences Between Low Versus High Skeletal Muscle Hypertrophic Responders to Resistance Exercise Training: Current Perspectives and Future Research Directions. Front. Physiol. 2018. 
Sahin K, Pala R, Tuzcu M, Ozdemir O, Orhan C, Sahin N, Juturu V (2016). Curcumin prevents muscle damage by regulating NF-kB and Nrf2 pathways and improves performance: an in vivo model. J Inflamm Res. 2016; 9: 147-154

Srichamroen A, Field C J, Thomson A B R, Basu T K (2008). The Modifying Effects of Galactomannan from Canadian-Grown Fenugreek (Trigonella foenum-graecum L.) on the Glycemic and Lipidemic Status in Rats. J Clin Biochem Nutr. 43(3): 167-174.

Takahashi M, Suzuki K, Kim H K, Otsuka Y, Imaizumi A, Miyashita A, Sakamoto S (2013) Effects of Curcumin Supplementation on Exercise- Induced Oxidative Stress in Humans. Int J Sports Med 2014; 35(06): 469-475

Weir J P (2005). Quantifying Test- Retest Reliability Using the Intraclass. Journal of Strength and Conditioning Research, 2005, 19(1), 231-240 2005

Westerblad H, Allen D G, Lannergren J (2002). Muscle fatigue: lactic acid or inorganic phosphate the major cause? News Physiol Sci 17:17-21

Zamora A J, Tessier F, Marconnet P, Margaritis I, Marini J F. (1995). Mitochondria changes in human muscle after prolonged exercise, endurance training and selenium supplementation. Eur J Appl Physiol Occup Physiol. 1995;71(6):505-11

Zuniga J M, Housh T J, Camic C L, Hendrix C R, Schmidt R J, Mielke M and Johnson G O (2010). A mechanomyographic fatigue threshold test for cycling Int. J. Sports Med. 31 $636-43$ 
VITA

1. Quinnipiac University, BS in Athletic Training

2. Lauren Herrick, BS, ATC 